\title{
Neutrophils responsive to endogenous IFN- $\beta$ regulate tumor angiogenesis and growth in a mouse tumor model
}

\author{
Jadwiga Jablonska, Sara Leschner, Kathrin Westphal, Stefan Lienenklaus, and Siegfried Weiss
}

Molecular Immunology, Helmholtz Centre for Infection Research, Braunschweig, Germany.

\begin{abstract}
Angiogenesis is a hallmark of malignant neoplasias, as the formation of new blood vessels is required for tumors to acquire oxygen and nutrients essential for their continued growth and metastasis. However, the signaling pathways leading to tumor vascularization are not fully understood. Here, using a transplantable mouse tumor model, we have demonstrated that endogenous IFN- $\beta$ inhibits tumor angiogenesis through repression of genes encoding proangiogenic and homing factors in tumor-infiltrating neutrophils. We determined that IFN- $\beta$-deficient mice injected with B16F10 melanoma or MCA205 fibrosarcoma cells developed faster-growing tumors with better-developed blood vessels than did syngeneic control mice. These tumors displayed enhanced infiltration by $\mathrm{CD} 11 \mathrm{~b}^{+} \mathrm{Gr}^{+}$neutrophils expressing elevated levels of the genes encoding the proangiogenic factors VEGF and MMP9 and the homing receptor CXCR4. They also expressed higher levels of the transcription factors c-myc and STAT3, known regulators of VEGF, MMP9, and CXCR4. In vitro, treatment of these tumor-infiltrating neutrophils with low levels of IFN- $\beta$ restored expression of proangiogenic factors to control levels. Moreover, depletion of these neutrophils inhibited tumor growth in both control and IFN- $\beta$-deficient mice. We therefore suggest that constitutively produced endogenous IFN- $\beta$ is an important mediator of innate tumor surveillance. Further, we believe our data help to explain the therapeutic effect of IFN treatment during the early stages of cancer development.
\end{abstract}

\section{Introduction}

Angiogenesis is one of the hallmarks of the development of malignant neoplasias. Primary tumors of a certain size and metastases require the growth of new blood vessels in order to be supplied with nutrients and oxygen (1-3). Accordingly, at a size of 1-2 $\mathrm{mm}^{3}$, tumors alter their angiogenic phenotype and support continuous proliferation of endothelial cells $(3,4)$. This angiogenic switch is activated by disruption of the balance between endogenous angiogenic stimulators and inhibitors. It leads to the uncontrolled growth of vessel mainly via stimulation of VEGF (5-7). However, factors and signaling pathways leading to tumor vascularization are not yet fully understood.

Infiltrating leukocytes, i.e., macrophages and neutrophils, were shown to be involved in tumor growth (8-11). Most likely, such cells are attracted to the cancerous tissue by chemotactic factors. The chemokine CXCL12 and the growth factor VEGF were suggested as such chemoattractants (12). CXCL12, a homeostatic chemokine, is produced constitutively in almost all organs (13). However, overexpression of CXCL12 and its receptor CXCR4 has been observed in many highly vascularized neoplasias (14-16). In agreement, expression of CXCL12 appears to be enhanced by hypoxia, as found in internal regions of large tumors (17).

In this study, we investigated the influence of IFN- $\beta$, a member of the family of type I IFNs, on the growth and angiogenesis of tumors. IFNs consist of 1 IFN- $\beta$ and 13 IFN- $\alpha$, all of which bind to a single receptor (IFNAR). A hierarchy of type I IFNs was shown to exist in many cell types. Upon stimulation, IFN- $\beta$ is responsible for triggering the cascade of IFN production via autocrine or para-

Conflict of interest: The authors have declared that no conflict of interest exists. Citation for this article: J Clin Invest. 2010;120(4):1151-1164. doi:10.1172/JCI37223. crine loops (18). In addition, IFN- $\beta$ was claimed to be expressed constitutively at low levels even in an uninduced state $(19,20)$. Thus, IFN- $\beta$ could influence cell differentiation and fate even under noninflammatory conditions.

Under inflammatory conditions, IFNs have been shown to exhibit diverse functions. For instance, they could act in an antiproliferative and immunoregulatory manner (21). These functions are of special interest for tumor therapy, since IFNs were shown to be involved in the elimination of immunogenic tumors (22). In addition, their antitumor potential is used for therapy of many human cancers (22, 23). However, the molecular mechanisms leading to therapeutic effects and the cells involved in it are still not entirely clear.

Using mice in which the gene for IFN- $\beta$ has been inactivated, we could establish that constitutive production of low amounts of this cytokine apparently slows the growth of B16F10 melanoma and MCA205 fibrosarcoma tumors. The effect is exerted by repressing myeloid cell-mediated angiogenesis. We could show that endogenous IFN- $\beta$ inhibits CXCR4 expression in neutrophils by downregulating Stat 3 and $c-m y c$. Moreover, IFN- $\beta$ inhibits VEGF expression in these cells. VEGF is the second most potent tumor chemoattractant for neutrophils. Lack of IFN- $\beta$ leads to reduced migration of CD $11 b^{+} \mathrm{Gr}-1^{+}$ neutrophils into the tumor, resulting in decreased angiogenesis and retardation of tumor growth. Taken together, our work strongly supports the concept that neutrophils not only play a role in host responses during infections, but are also required for tumor growth and angiogenesis. Constitutive production of low amounts of IFN- $\beta$ counteracts their cancer-supportive function.

\section{Results}

Enhanced growth of B16F10 melanoma in IFN- $\beta$-deficient mice. Type I IFNs, when given exogenously to tumor patients, retard tumor 


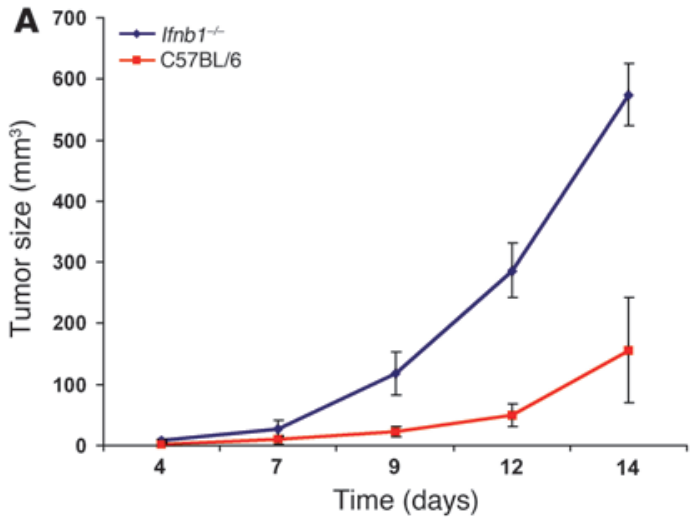

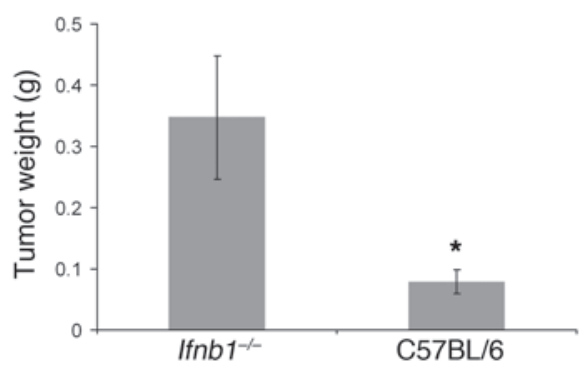

B
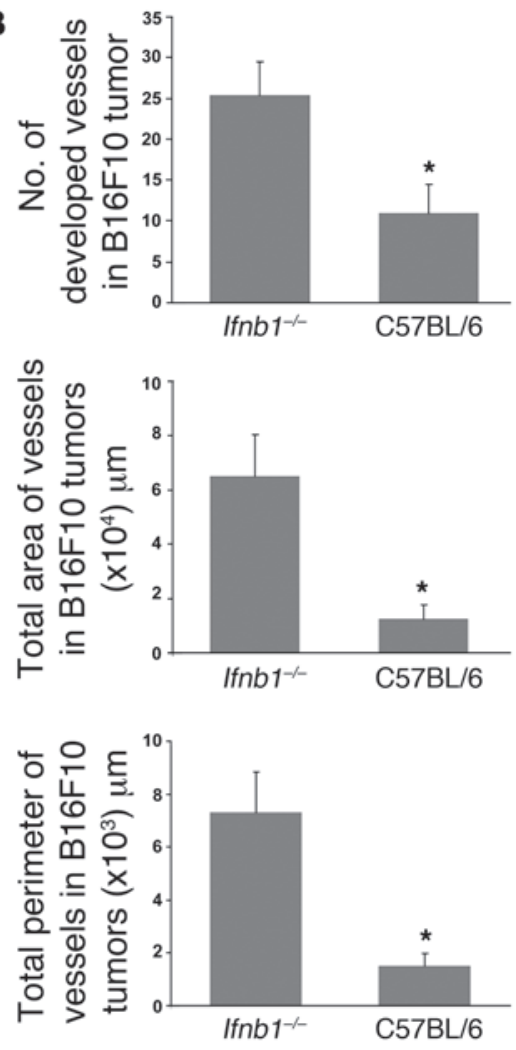

c
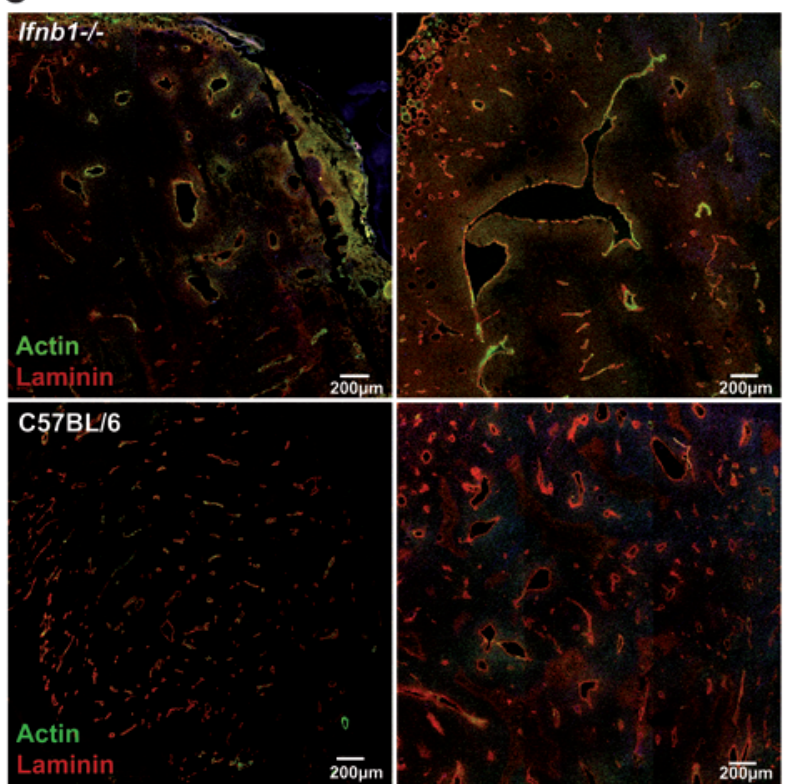
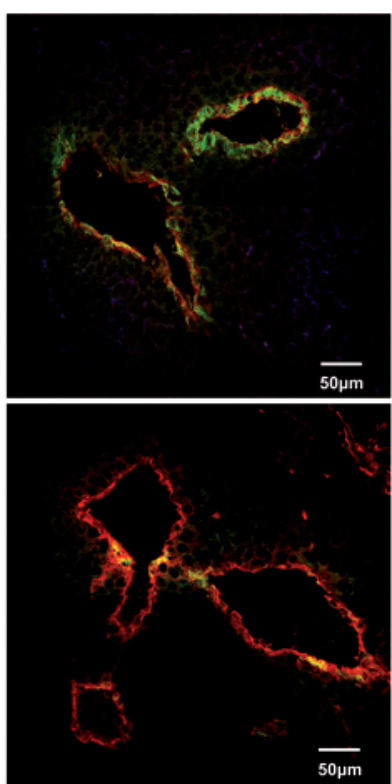

Figure 1

Enhanced tumor growth and angiogenesis in Ifnb1-/- mice. (A) Growth and size of tumors are significantly higher in Ifnb1 $1^{-/}$mice. B16F10 melanoma cells were injected s.c. into the abdomen of C57BL/6 or Ifnb $1^{-1-}$ mice, and tumor growth was monitored. At day 14 , mice were sacrificed and tumor weight and diameter were measured. Experiments were done with at least 5 animals per group and repeated at least 3 times with similar results. Data represent mean \pm SEM. ${ }^{*} P \leq 0.01$. (B) The number, the area, and the perimeter of vessels in tumors isolated from Ifnb $1^{-/-}$mice are significantly higher than in C57BL/6 mice. For histological analysis, material was collected as described in A. $10 \mu \mathrm{m}$ cryosections were prepared and stained for laminin and actin (SMA). Fully developed vessels (laminin ${ }^{+}$actin $^{+}$) were counted and the size of vessels calculated using ImageJ software. Histograms represent data from at least 3 independent experiments with at least 3 mice per group. More than 20 fields of view were analyzed in each experiment. Data represent mean \pm SEM. ${ }^{*} P \leq 0.01$. (C) B16F10 tumors grown in Ifnb1-/- mice exhibit a higher content of fully developed vessels (laminin ${ }^{+} a$ tin $^{+}$). Histological analysis was done with material collected as described in $\mathbf{A}$, with $10 \mu \mathrm{m}$ cryosections prepared and stained for laminin (red) and actin (green). Scale bars: $200 \mu \mathrm{m}$ and $50 \mu \mathrm{m}$, respectively. Photographs represent data from at least 3 independent experiments, with at least 3 mice per group. growth $(24,25)$. Similarly, mice are protected when challenged with tumor cells that produce IFN as recombinant protein $(26,27)$. However, there is still a question of how important endogenous type I IFN is for tumor development and editing. Therefore, we employed a transplantable murine tumor model as first approximation to answer this question. Mice that are deficient for IFN- $\beta$ were tested, since this isotype has a unique role among the type I IFNs.
We challenged C57BL/6 mice and syngeneic Ifnb1/-/- mice s.c. with the melanoma cell line B16F10 and followed tumor growth for 14 days (Figure 1A). After this time, mice were sacrificed and tumor weight was determined. Notably, tumors of Ifnb $1^{-/-}$mice grew faster. They reached larger sizes and higher weights compared with tumors of control mice (Figure 1A).

Direct antiproliferative effects of IFN- $\beta$ on B16F10 melanoma cells could be the reason for the increased growth of tumors in 
A
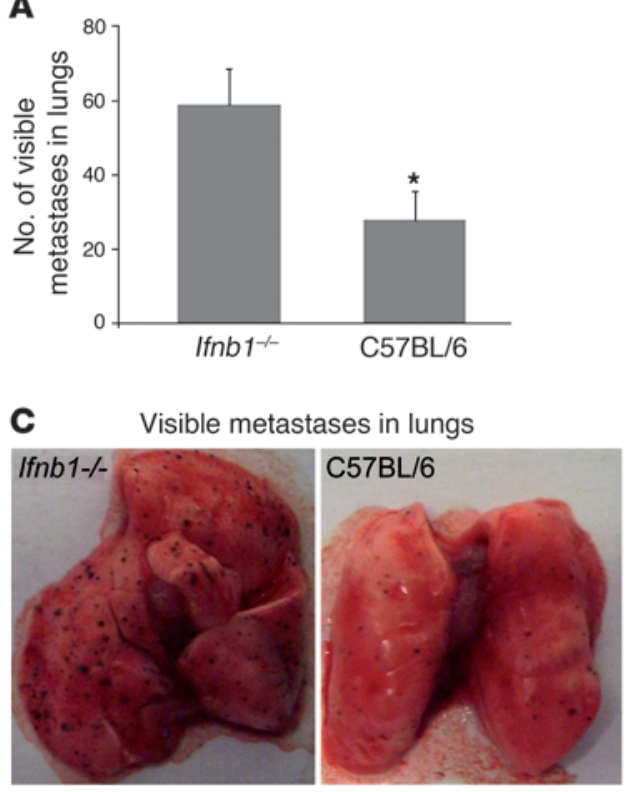

B
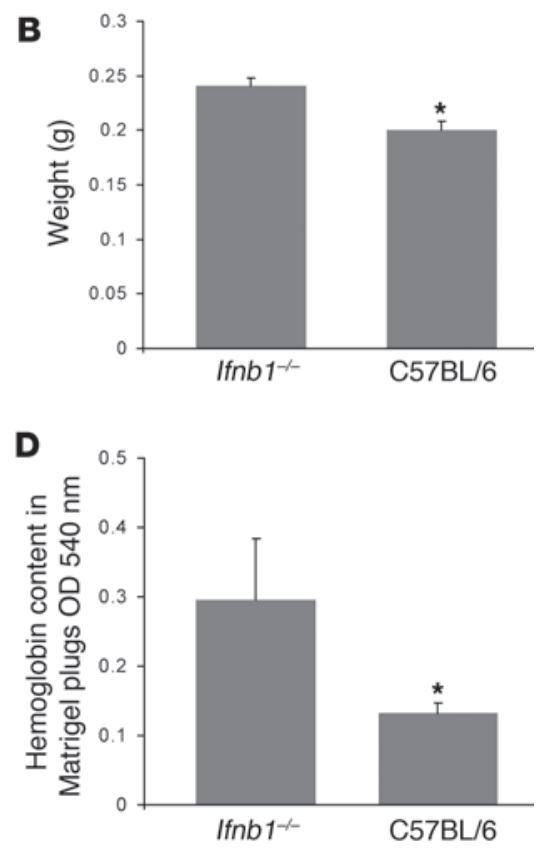

Figure 2

Increased numbers of lung metastases and enhanced Matrigel angiogenesis in Ifnb $1^{-/}$ mice. (A-C) Increased formation of metastases in lungs of Ifnb1 $1^{-/-}$mice. Ifnb $1^{-/-}$and control mice were injected i.v. with $4 \times 10^{5}$ B16F10 melanoma cells. Mice were sacrificed after 14 days; lungs were removed and analyzed by counting visible B $16 \mathrm{~F} 10$ colonies and determining the weight of the lungs. (A) Number of visible colonies in lungs of $/ \mathrm{fnh}^{-/-}$and $\mathrm{C} 57 \mathrm{BL} / 6$ mice. (B) Weight of lungs isolated from Ifnb $1^{-/-}$and C57BL/6 mice. (C) Macroscopic comparison of lungs with B16F10 colonies from Ifnb1-/and C57BL/6 mice. (D) In vivo angiogenesis assay shows higher vessel development in plugs isolated from Ifnb1 $1^{-1-}$ mice. BD Matrigel was injected s.c. into the abdomen of mice. At day 10, plugs were removed and analyzed for hemoglobin content using Drabkin reagent. Experiments were done twice, with at least 5 animals per group. Data represent mean \pm SEM. ${ }^{*} P \leq 0.01$.
Ifn $b 1^{-/-}$mice. To rule out this possibility, we used Ifnar $1^{-/-}$animals. In such mice, IFN- $\beta$ is produced, but the recipient cells cannot respond because the receptor is deleted. In contrast, tumor cells will remain responsive to IFN- $\beta$. In such mice, B16F10 tumor growth was also faster than in control mice, comparable with the results obtained for Ifnb $1^{-/-}$mice. Thus, a direct antiproliferative effect of IFN- $\beta$ on the melanoma cells could be excluded (Supplemental Figure 5; supplemental material available online with this article; doi:10.1172/JCI37223DS1).

Enhanced tumor angiogenesis in IFN- $\beta$-deficient mice. Tumor development depends strongly on angiogenesis. Without development of functional vessels, tumor growth stops at 1-2 mm diameter (1). We therefore tested the status of blood vessels in tumors growing in Ifn $b^{-/-}$and normal mice by histology. Tumors of comparable size were taken for analysis to exclude size-dependent differential hypoxic conditions. Interestingly, the number of vessels as well as the area covered by vessels and their perimeter were significantly larger in Ifnt $b^{-/-}$mice compared with controls (Figure 1B).

Functional blood vessels consist of 3 layers: smooth muscle cells, the endothelium, and the lumen. Especially important is the muscle cell layer, since it regulates the blood flow. Blood vessels of tumors often lack the smooth muscle layer, which impairs their ability to control blood flow (28). Therefore, we examined tumor sections from normal and $I f n b^{-/-}$mice for $\alpha$ SMA as a marker for smooth muscle cells and counterstained for laminin as a marker for endothelial cells. As shown in Figure 1C, vessel development of tumors grown in Ifnb $\mathrm{b}^{-/}$mice was much more advanced. Stronger signals were obtained for $\alpha$ SMA as compared with vessels from control tumors. The lower actin signal found in such control tumors suggests an immature phenotype and could explain the smaller size of tumors from normal mice.

Absence of endogenous IFN- $\beta$ enhances metastasis formation in the lung. Formation of metastasis is another hallmark of malignant tumors. Cancer cells spread from the primary tumor via the bloodstream to grow at distant sites. Often this is the cause of death of tumor patients (16). It is generally accepted that metastasis formation depends on efficient angiogenesis (29). Factors involved in vessel formation, such as VEGF, provide signals for chemotaxis and motility of tumor cells to develop secondary, metastatic tumors in various organs. For that reason, we were interested in whether increased vessel formation observed in Ifnt-/- mice would also influence the metastasis process. Thus, we injected B16F10 melanoma cells i.v. into I $\mathrm{fn}^{-/-}$and control mice. After 14 days, the animals were sacrificed, lungs were isolated, and formation of visible B16F10 tumor colonies was quantified as an indicator for metastasis formation. Higher numbers of visible tumor cell colonies with increased size were observed in the lungs of Ifn $b^{-/-}$mice compared with normal mice (Figure 2, A and B). In addition, due to the increased metastatic burden, the lungs of Ifnb-/- animals displayed significantly higher weight than lungs from normal mice (Figure 2C).

Absence of endogenous IFN- $\beta$ enhances vessel formation in an in vivo angiogenesis assay. To see whether the difference in induced angiogenesis between Ifnb-/- and control mice occurred only in a tumor situation or might be a more general phenomenon, we s.c. injected Matrigel containing VEGF into both types of mice and analyzed vessel formation in such pads. Since the hemoglobin content in the pad is directly correlated with the number of vessels, we determined the concentration of hemoglobin using Drabkin reagent as readout. As shown in Figure 2D, also in this case, the number of vessels formed is higher in mice deficient for IFN- $\beta$ compared with WT mice. Thus, increased angiogenesis in the absence of endogenous IFN- $\beta$ is not restricted to tumors.

Augmented neutrophil numbers in B16F10 tumors of Ifnb $b^{-/-}$mice. The tumor environment is largely orchestrated by inflammatory myeloid cells such as neutrophils and macrophages (30). Moreover, it was postulated that therapeutically administered type I IFN mediates its antitumor effects mainly by acting on hematopoietic cells of the host (22). Therefore, we analyzed the content of myeloid cells populating tumors that were grown in normal mice or mice lacking IFN- $\beta$. Interestingly, large differences were observed in the number of the CD $11 \mathrm{~b}^{+} \mathrm{Ly} 6 \mathrm{G}(\mathrm{Gr} 1)^{+} \mathrm{Ly}_{6 \mathrm{C}^{-}}$int tumor-infiltrating neutrophils (characterized in Supplemental Figure 1) between 
A

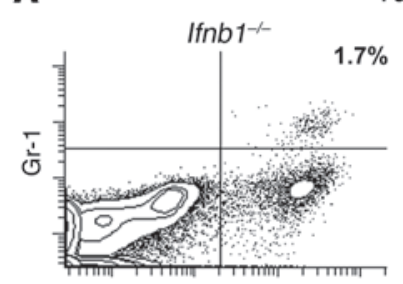

CD11b

\section{C}
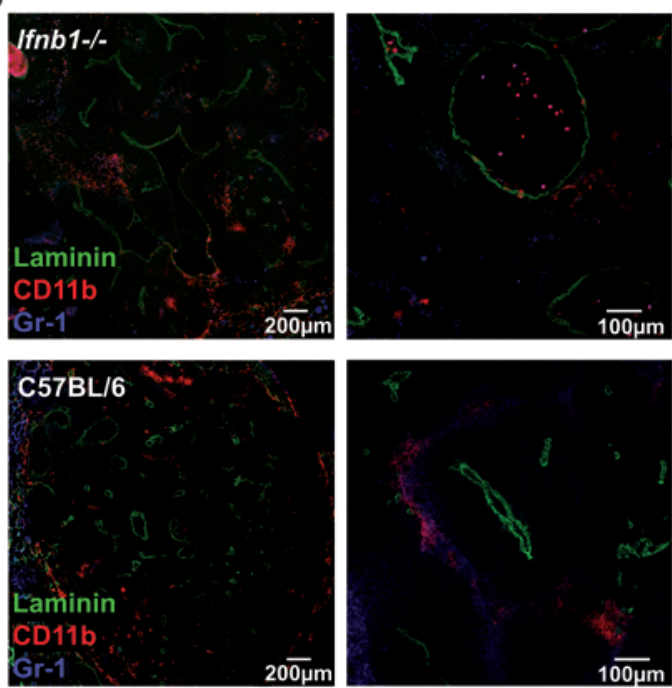

Tumor

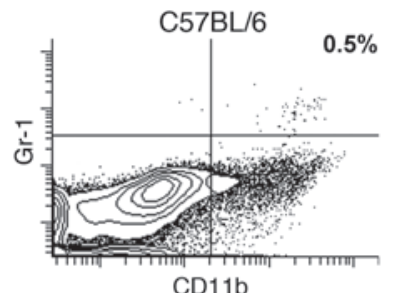

CD11b
B

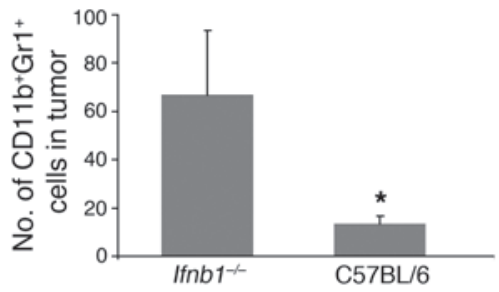

Bone marrow

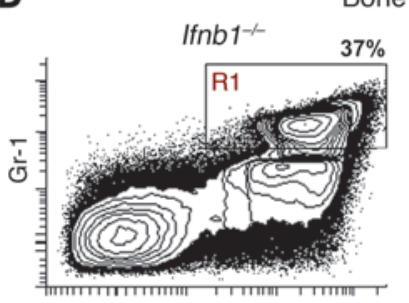

CD11b
D
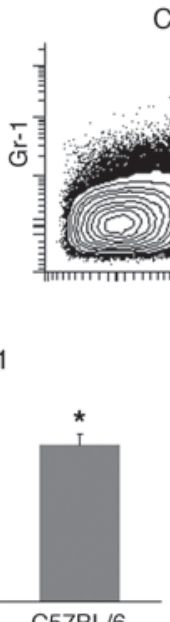

C57BL/6

R1
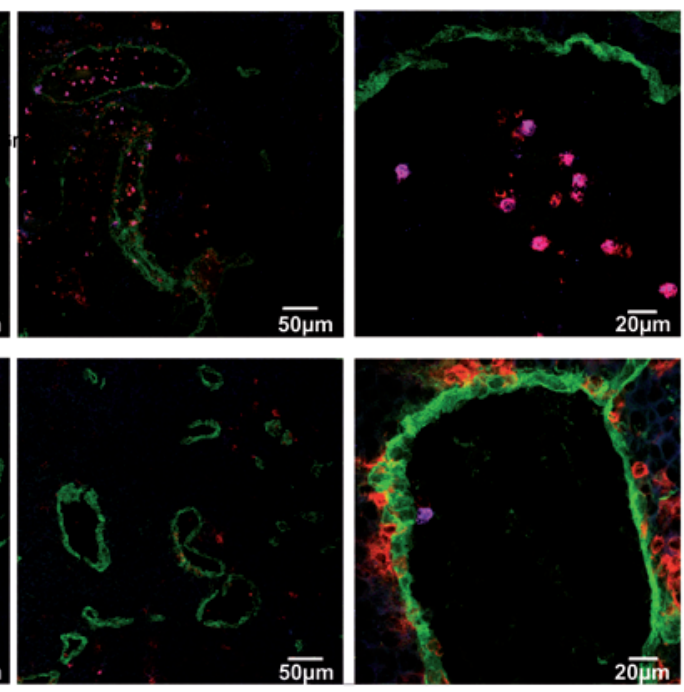

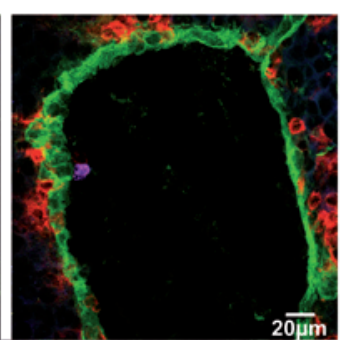

Blood

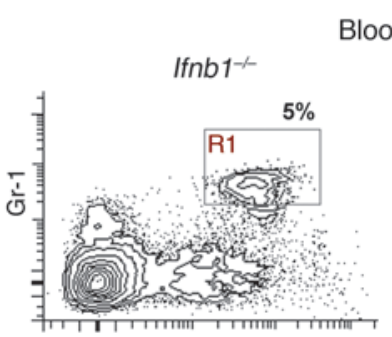

CD11b

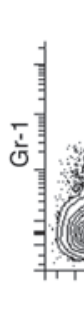

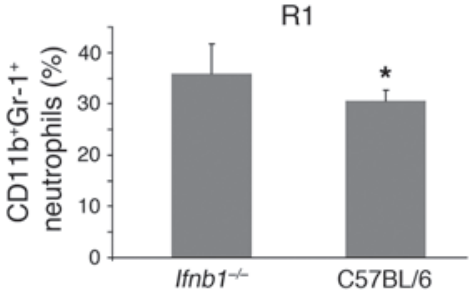

C57BL/6

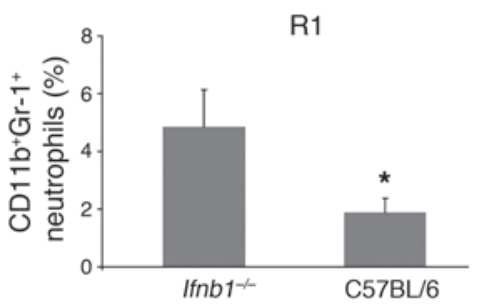

Figure 3

Enhanced numbers of CD11 b+Gr-1+ neutrophils in tumors of Ifnb1-/- mice. (A) Percentage of CD11 b+Gr-1+ cells (characterized in more detail in Supplemental Figure 1) in tumor is altered in Ifnb1-/ mice compared with C57BL/6 mice. Flow cytometry of myeloid cells from tumors of Ifnb $1^{-1-}$ and C57BL/6 mice. Tumors were removed at day 14; single-cell solutions were prepared, stained, and analyzed using the BD LSR II System. Data were analyzed with FACSDiva software. (B and C) Number of myeloid cells in tumors isolated from Ifnb1 ${ }^{-l-}$ mice is higher than in C57BL/6 mice. Tumors were removed, and $10 \mu \mathrm{m}$ cryosections were prepared and stained for Gr-1 (blue), CD11b (red), and laminin (green). Number of neutrophils was calculated per 20 fields of view. Experiments were repeated at least twice, with at least 5 animals per group. Scale bars: $200 \mu \mathrm{m}, 100 \mu \mathrm{m}, 50 \mu \mathrm{m}$, and $20 \mu \mathrm{m}$ respectively. (D) Percentage and number of CD11b+Gr-1+ neutrophils in bone marrow and blood are altered in Ifnb $1^{-/-}$mice compared with C57BL/6 mice. Experiments were done twice with at least 5 animals per group. Data represent mean \pm SEM. ${ }^{*} P \leq 0.01$. 
the 2 types of mice. Tumors isolated from Ifn $b^{-/-}$mice exhibited significantly higher contents of neutrophils than tumors from normal mice (Figure 3, A-C). These cells accumulate especially in close vicinity to vessels or in their lumen (Figure 3C).

Since in the tumors many different populations of cells with hematopoietic origin could be observed, the characteristic of tumor-infiltrating neutrophils was confirmed by additional staining. Diverse myeloid cell markers were employed to distinguish such cells from phenotypically similar inflammatory monocytes $\left(\right.$ Ly6C $\mathrm{C}^{+} \mathrm{Ly} 6 \mathrm{G}^{+}$) (31), tumor-associated monocytic cells (TAMs; CD68 $\left.{ }^{+} \mathrm{F} 4 / 80^{+}\right)(32-34)$, or myeloid suppressor cells $\left(\mathrm{Ly}_{6} \mathrm{C}^{+} \mathrm{Ly}_{6 \mathrm{G}} \mathrm{CD}^{+} 11 \mathrm{~b}^{+}\right)(35)$ that might be observed in tumors as well. This analysis revealed that the population of neutrophils was homogeneous and did not contain populations of cells mentioned above, i.e., cells were CD $11 \mathrm{~b}^{+} \mathrm{CD} 11 \mathrm{c}^{-} \mathrm{Ly} \mathrm{C}^{-}{ }^{- \text {int }} \mathrm{Ly} 6 \mathrm{G}^{+} \mathrm{F} 4 / 80^{-} \mathrm{CD} 68^{-}$ (Supplemental Figure 1).

The differential numbers of neutrophils in tumors of both types of mice prompted us to analyze the number of neutrophils in bone marrow and blood isolated from such tumor-bearing mice. Interestingly, only a small difference could be revealed in the bone marrow, with the content of $\mathrm{CD} 11 \mathrm{~b}^{+} \mathrm{Gr} 1^{+}$neutrophils being slightly higher in Ifn $b^{-/}$than in normal mice (Figure 3D). In the blood, however, the percentage of neutrophils was notably increased in tumor-bearing Ifn $b^{-/-}$mice (Figure 3D and Supplemental Figure 2), suggesting altered migratory capacities of these cells. No difference could be detected between the 2 types of mice when they did not bear a tumor. This indicates that the tumor cells might induce the recruitment of $\mathrm{CD}_{11} \mathrm{~b}^{+} \mathrm{Gr} 1^{+}$neutrophils (Supplemental Figure 2) via cytokines and chemotactic factors.

Efficient tumor angiogenesis depends on infiltrating neutrophils. Myeloid cells were suggested as playing a role in the initial stage of tumor vessel development (10). Therefore, we wanted to examine the function of such cells in growth and vascularization of B16F10 tumors. We depleted such cells from normal and Ifnt-/mice using anti-Gr1 antibodies and then injected B16F10 cells s.c. Anti-Gr1 antibodies depleted in our hands only the neutrophil population $\left(\mathrm{CD}_{1} 1 \mathrm{~b}^{+} \mathrm{Gr} 1^{+}\right)$without influencing DCs, monocytes, and macrophages (see Supplemental Figure 3 for effective depletion of $\mathrm{Gr}^{+}$cells). Tumors were removed after 14 days, and their weights were determined. Mice depleted of granulocytes showed reduced tumor growth. Interestingly, the difference between control and $\mathrm{In}^{-1-}$ mice was no longer observed when mice were treated with anti-Gr1 antibodies (Figure 4A).

Analysis of the vessel status of such tumors by histology revealed significantly lower vessel development in anti-Gr1-treated mice, independent of whether they were IFN- $\beta$ deficient or WT (Figure 4B). Similarly, according to the smooth muscle actin content, vessels were less differentiated in tumors from mice treated with anti-Gr1 independent of the presence of endogenous IFN- $\beta$ (Figure 4C).

$C D 11 b^{+} G r 1^{+}$neutrophils supporting tumor angiogenesis are essential targets of endogenous type I IFN in vivo. We wanted to unambiguously confirm that neutrophils, which under normal circumstances support tumor angiogenesis, are subdued by endogenous IFN- $\beta$. To this end, we mixed B16F10 cells with neutrophils that were isolated from B16F10 tumors growing in either Ifnar $1^{-/-}$or normal mice and injected the mixture s.c. into normal mice according to Yang et al. (36). In this situation, the only cell population that lacks responsiveness to endogenous IFN is the angiogenesis-supporting neutrophils from Ifnar $1^{-/-}$mice. To increase the effect, sorted neutrophils from tumors of either mouse were additionally injected i.v. on days 2 and 4. Tumor growth was monitored for 14 days; then tumors were removed, weighed, and additionally analyzed by immunohistology. A dramatic increase in tumor growth and weight was observed in mice in which tumor angiogenesis was supported by neutrophils isolated from tumors of Ifnar $1^{-/-}$mice (Figure 4D). This was also reflected by the maturation state of the vessels in such mice. Vessels from tumors supported by neutrophils isolated from tumors of Ifnar $1^{-/-}$mice were highly positive for smooth muscle actin, indicating mature vessels (Figure 4E). In contrast, in tumors in which angiogenesis was supported by neutrophils of normal mice, hardly any actin staining could be detected. This compellingly demonstrates that the essential cell population responsible for the excessive angiogenesis in mice with a defective IFN system is that of neutrophils.

IFN- $\beta$ regulates expression of homing and angiogenic factors in neutrophils. Migration of $\mathrm{CD} 11 \mathrm{~b}^{+} \mathrm{Gr} 1^{+}$neutrophils into tumors appeared to be important for tumor growth. Therefore, we examined chemokine receptors that might be responsible for differences in the number of tumor-infiltrating neutrophils between control and Ifnb $1^{-1-}$ mice. A significant difference was found in the percentage of CXCR4 ${ }^{+}$neutrophils isolated from blood and tumor (Figure 5, A and B). This was especially apparent in blood. No difference was found for other members of the homeostatic chemokine receptors such as CCR7 (data not shown). CXCR4 is known to be a potent chemokine receptor directing cells into several organs but also into tumors $(16,30,37)$. Its ligand CXCL12 has been found in increased amounts in many tumors (15), and it is supposed to be upregulated by hypoxia. Under our experimental conditions, amounts of CXCL12 were indeed significantly upregulated in tumors isolated from Ifnb $b^{-1-}$ compared with normal mice (Supplemental Figure 4).

Consequently, we wanted to see the influence of IFN- $\beta$ on expression of homing and angiogenesis factors in tumor-infiltrating neutrophils. To this end, we sorted such cells from B16F10 melanoma tumors and isolated RNA. As judged by quantitative RT-PCR (qRT-PCR), several angiogenic factor genes such as Ang1, Ang2, and Pecam showed comparable expression levels in neutrophils of normal and Ifnb1 $1^{-/-}$mice (data not shown). However, important differences were found for expression of Cxcr4, Vegf, and Mmp9 that were strongly reduced in neutrophils from tumors of normal mice. MMP9 is a proteolytic enzyme, which is known to promote angiogenesis and vasculogenesis by increasing availability of VEGF in tumors and endothelial cells $(10,36,38)$. VEGF, in addition to its role as a main angiogenesis stimulator, has also been shown to be important in recruiting myeloid cells into tumors $(12,39,40)$.

To prove the direct influence of IFN- $\beta$ on regulation of these proangiogenic factors, we isolated tumor-infiltrating $\mathrm{CD} 11 \mathrm{~b}^{+} \mathrm{Gr} 1^{+}$ neutrophils from Ifnb1 $1^{-/-}$mice (Supplemental Figure 7) and treated them with recombinant mouse IFN- $\beta$. In agreement with our working hypothesis, Cxcr4 as well as Vegf and Mmp9 were strongly downregulated upon IFN- $\beta$ treatment (Figure 5C).

Stat 3 and c-myc are downregulated by IFN- $\beta$. It is well established that IFN- $\beta$ downregulates $c-m y c$ expression (41-43). Growth and cell proliferation are influenced by $c-m y c$, but it is also known to regulate Cxcr4 $(44,45)$. Therefore, we established the expression pattern of $c-m y c$ in the tumor-infiltrating $\mathrm{CD} 11 \mathrm{~b}^{+} \mathrm{Gr} 1^{+}$neutrophils isolated from normal and If $b 1^{-/-}$mice. Interestingly, these cells obtained from tumors of Ifnb $1^{-/-}$mice exhibited higher $c-m y c$ expression than equivalent cells from tumors of normal mice. As above, treatment with exogenous IFN- $\beta$ decreased the expression of $c$-myc to levels found in cells from normal mice (Figure 5D). 

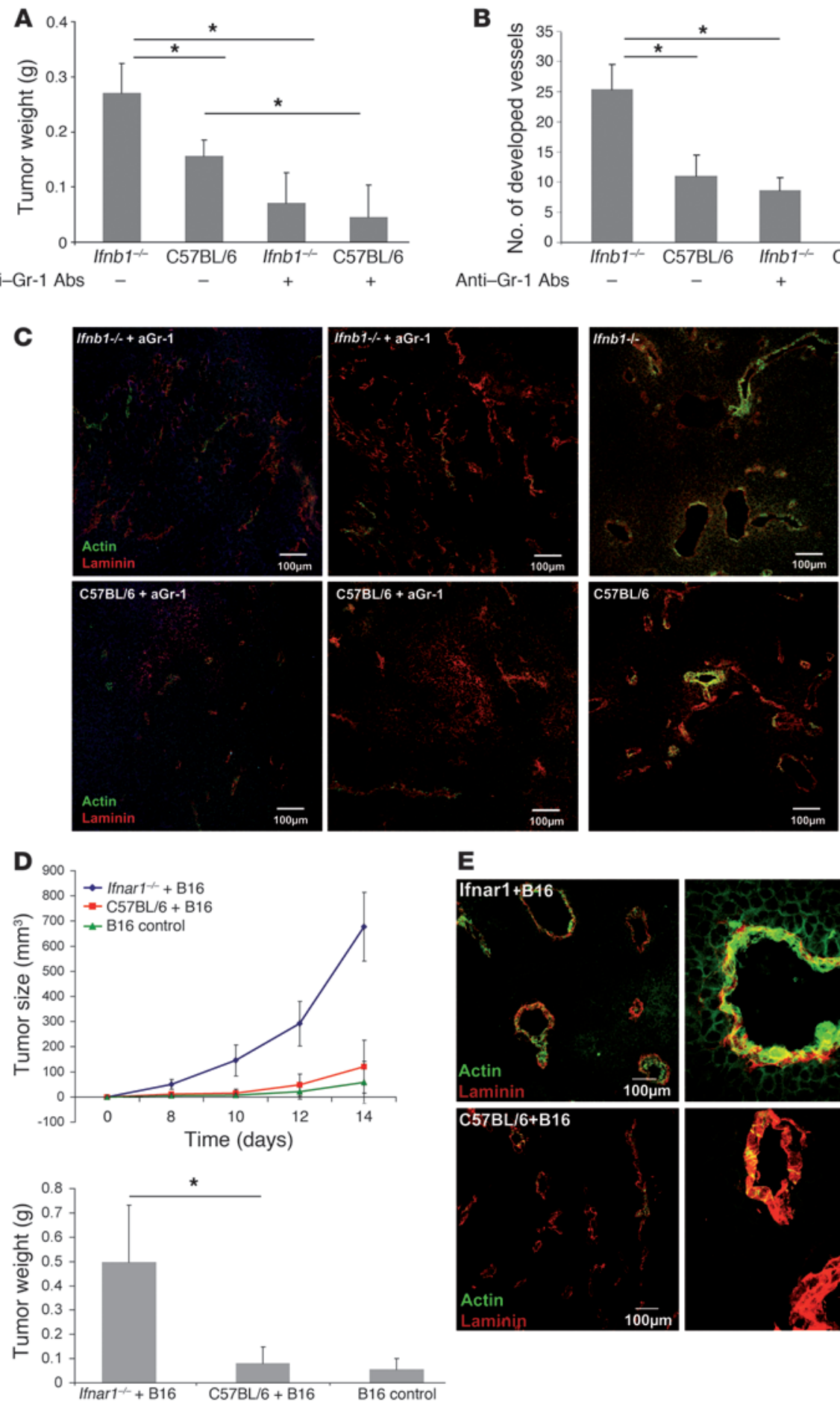

E
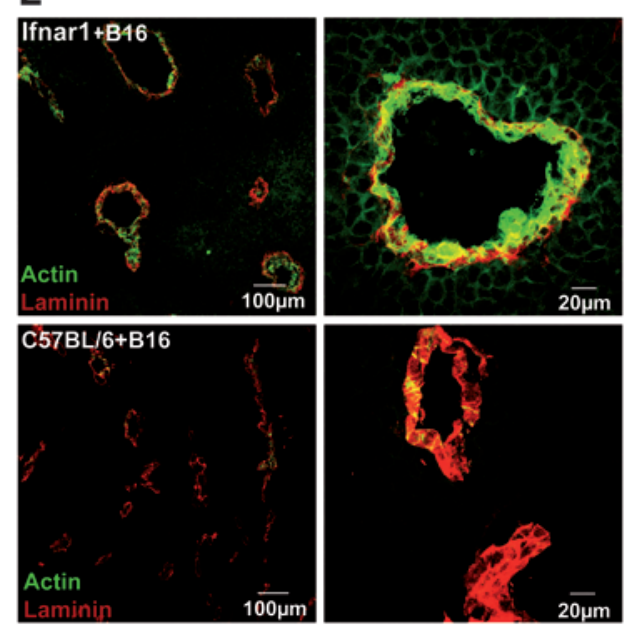

\section{Figure 4}

Essential role of IFN- $\beta$-responsive $\mathrm{CD} 11 \mathrm{~b}+\mathrm{Gr} 1+$ neutrophils in B16F10 tumor growth and angiogenesis. CD11b+Gr1+ cells were depleted by treatment with anti-Gr1 Ab, B16F10 cells injected s.c., and after 14 days, mice were sacrificed and tumors were removed, their weight determined, and cryosections stained for confocal microscopy. (A) Reduced tumor growth in mice depleted of CD11b+Gr1+ cells compared with untreated animals. (B and C) Number of developed vessels is reduced after depletion of $\mathrm{Gr}^{1+}$ cells. (D) Enhanced tumor growth depends strictly on type I IFN-reactive CD11b+Gr1+ neutrophils. Mice injected s.c. with B16F10 cells mixed with neutrophils obtained from tumor-bearing Ifnar1-1- mice (Ifnar $\left.{ }^{-1}+\mathrm{B} 16\right)$ show increased tumor development compared with mice injected with neutrophils obtained from tumorbearing WT mice (C57BL/6+B16) or B16 alone (B16 control). Experiments were carried out twice with at least 5 animals per group. Data represent mean \pm SEM. ${ }^{\star} P \leq 0.01$. Histology shows representative pictures. At least 20 fields of view were analyzed. (E) B16F10 tumors coinjected with neutrophils obtained from tumor-bearing Ifnar $1^{-/-}$mice (Ifnar-1-+B16) show a higher content of fully developed vessels (actin+laminin+) compared with B16F10 injected together with neutrophils obtained from tumor-bearing control mice (C57BL/6+B16).
STAT3 is known to be required for full activation of the Cxcr4 gene $(46,47)$. Consistent with the above results, qRT-PCR showed increased transcription of Stat 3 in tumor-infiltrating $\mathrm{CD} 11 \mathrm{~b}^{+} \mathrm{Gr} 1^{+}$ neutrophils from Ifnb1-/- mice (Figure 5D). Treatment of these cells with exogenous IFN- $\beta$ in vitro resulted in downregulation of Stat 3 transcription. This underscores the regulatory role that this cytokine plays at multiple steps of CXCR4 expression.

Independent of transcriptional regulation, STAT3 is activated by phosphorylation. We therefore stained blood and tumor resi- dent neutrophils of normal and Ifn $b^{-/-}$mice intracellularly with an antibody that is specific for phosphorylated Y705 of STAT3. As expected, neutrophils from Ifnb $1^{-/-}$mice expressed higher levels of pY705 STAT3 both in tumor-infiltrating neutrophils and neutrophils isolated from blood compared with the same cell population from normal mice (Figure 5E).

Endogenous IFN- $\beta$ influences the cytokine and chemokine milieu in tumors. Differential development of tumors and altered migratory capacities of myeloid cells depend upon differentially regulated 
A

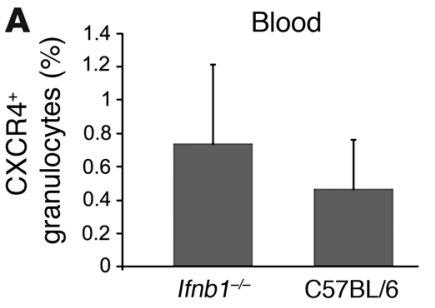

C

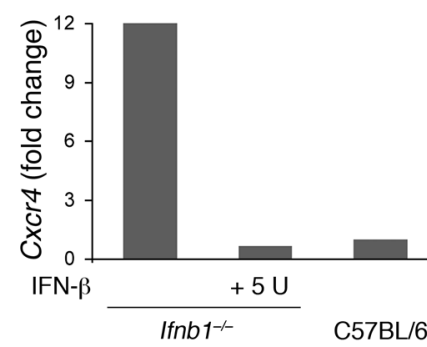

D

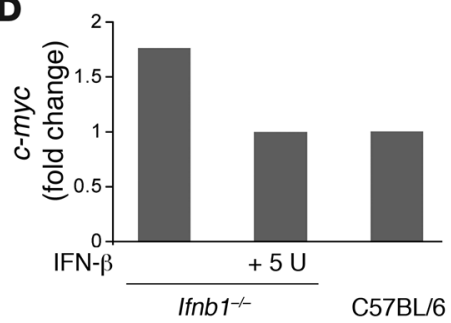

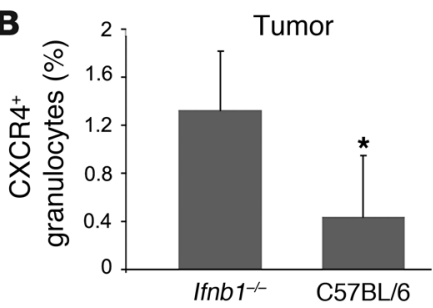
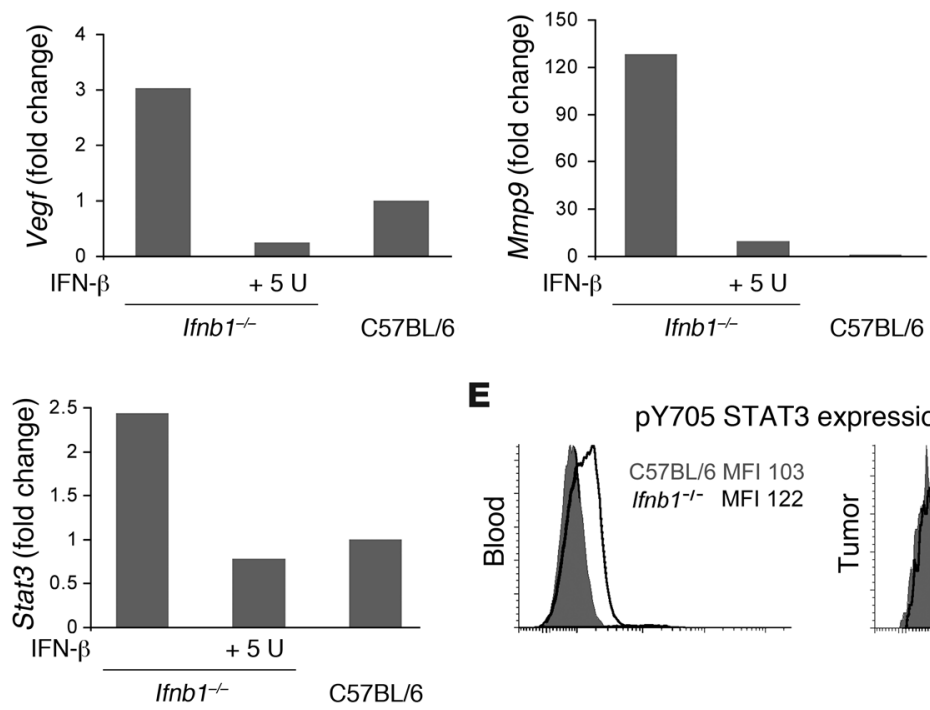

$\mathbf{E}$

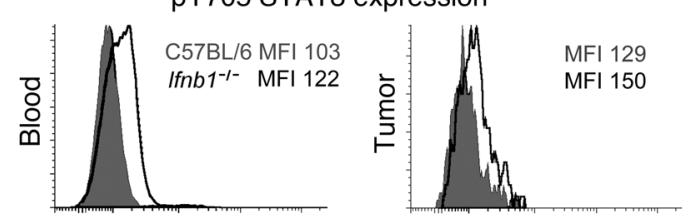

Figure 5

Altered characteristics of $\mathrm{CD} 11 \mathrm{~b}+\mathrm{Gr} 1^{+}$neutrophils isolated from Ifnb1-/- mice. (A) Percentage of $\mathrm{CXCR} 4^{+}$neutrophils in blood of tumor-bearing Ifnb $1^{-1-}$ and C57BL/6 mice. (B) Percentage of CXCR4 $4^{+}$neutrophils isolated from tumors of Ifnb $1^{-/-}$and C57BL/6 mice. Tumors were removed; single-cell solutions were prepared, stained, and analyzed using the BD LSR II System. Data were analyzed with FACSDiva software. Experiments were done twice with at least 5 animals per group. Data represent mean \pm SEM. ${ }^{*} P \leq 0.01$. (C) IFN- $\beta$ treatment downregulates Vegf, $M m p 9$, and Cxcr4 gene expression. Tumors were removed; single-cell solutions were prepared and stained, and CD11 $+\mathrm{br} 1+n e u t r o p h i l s$ were isolated. Monolayers of such cells were incubated with $5 \mathrm{U}$ rmIFN- $\beta$; after 4 hours, RNA was isolated, cDNA prepared, and gene expression measured using real-time RT-PCR. Cells were derived from 5 pooled tumors. All experiments were repeated at least 2 times. (D) High expression of $C x c r 4$ correlates with higher expression of $c-m y c$ and Stat 3 , and rmIFN- $\beta$ downregulates both $c-m y c$ and Stat 3 . CD11b+Gr-1+neutrophils were sorted from tumors and placed in culture with $5 \mathrm{U}$ rmIFN- $\beta$. After 4 hours, cDNA was prepared as described in $\mathbf{C}$. Cells were sorted from 5 pooled tumors. All experiments were repeated at least 2 times. (E) Expression of intracellular pSTAT3 in blood and tumor-infiltrating neutrophils was compared between control and Ifnb $1^{-1-}$ mice. Cell suspensions from blood and tumors were prepared, stained, and analyzed using the BD LSR II System. Data were analyzed with FACSDiva software. Experiments were done twice with at least 5 animals per group.

cytokines and chemokines $(48,49)$. Therefore, we tested the content of several cytokines in tumors isolated from Ifnb1 $1^{-/-}$and control mice. Tumors were homogenized and centrifuged, and supernatants were tested. Interestingly, not only homeostatic cytokines like CXCL12 were increased in tumors from Ifn $b^{-/-}$mice but also some proinflammatory mediators like IL- 6 or the inflammatory chemokine CCL2 (Supplemental Figure 4). The higher concentration of such cytokines might additionally explain the higher

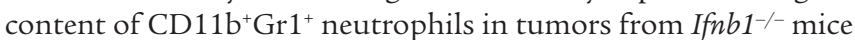
and their augmented growth. CCL2 is additionally known to be a potent chemoattractant for myeloid cells, such as monocytes and neutrophils $(39,50-54)$. It is believed to be produced by various cells, including vascular smooth muscle cells $(55,56)$. This may explain the higher content of this chemokine in the highly vascularized melanoma tumors from Ifnb $1^{-/-}$mice. In addition, the increased expression of IL- 6 in Ifnb $1^{-/-}$mice might have an impact on survival and proliferation of $\mathrm{CD} 11 \mathrm{~b}^{+} \mathrm{Gr} 1^{+}$neutrophils in the tumor, as described $(57,58)$.
Retardation of tumor growth in IFN- $\beta$-sufficient mice is independent of $T, B$, and NK cells. Type I IFNs have been shown to influence the activity of T and B cells $(21,59)$. To exclude the involvement of these cells in the inhibition of tumor growth in normal mice, we repeated our experiment using Rag $2^{-/-}$and Ifnb $1^{-/-}$Rag $2^{-/-}$mice, which lack $\mathrm{T}$ and $\mathrm{B}$ lymphocytes. B16F10 melanoma cells were injected s.c. into these mice, and tumor growth was monitored for 14 days. At day 14 after infection, tumor size and weight were determined. Tumors grew much faster in Ifnb $1^{-/-}$Rag2 $2^{-/-}$mice and reached larger sizes than tumors from Rag2 $2^{-/-}$control mice (Figure 6A). Numbers of tumor-infiltrating $\mathrm{CD} 11 \mathrm{~b}^{+} \mathrm{Gr} 1^{+}$neutrophils were also significantly

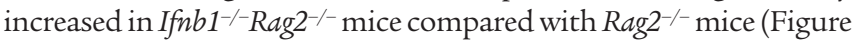
6 , B-D). Moreover, angiogenic processes were also more advanced in animals lacking IFN- $\beta$ (Figure $6 \mathrm{E}$ ). Thus, neither T nor B lymphocytes are involved in the IFN- $\beta$-dependent restriction of tumor growth and tumor angiogenesis in normal mice.

RAG-deficient mice still contain NK cells that could be involved in the retarded tumor growth. NK cells are known to be responsive 
A ${ }^{700}$ B
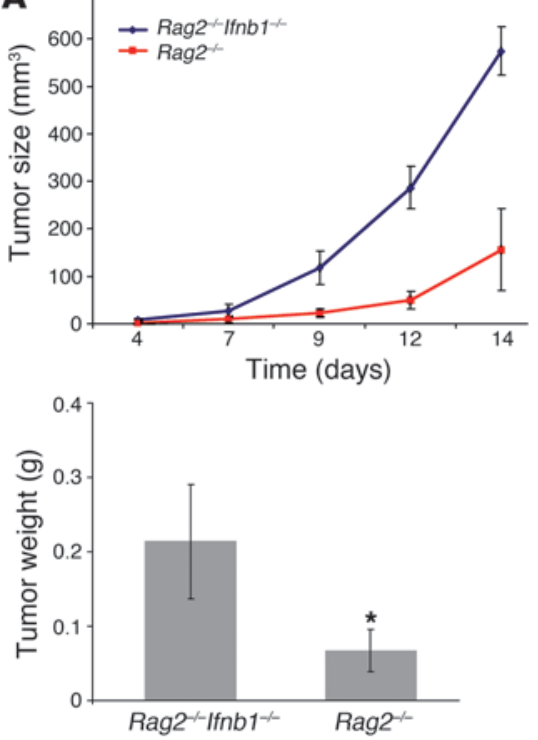
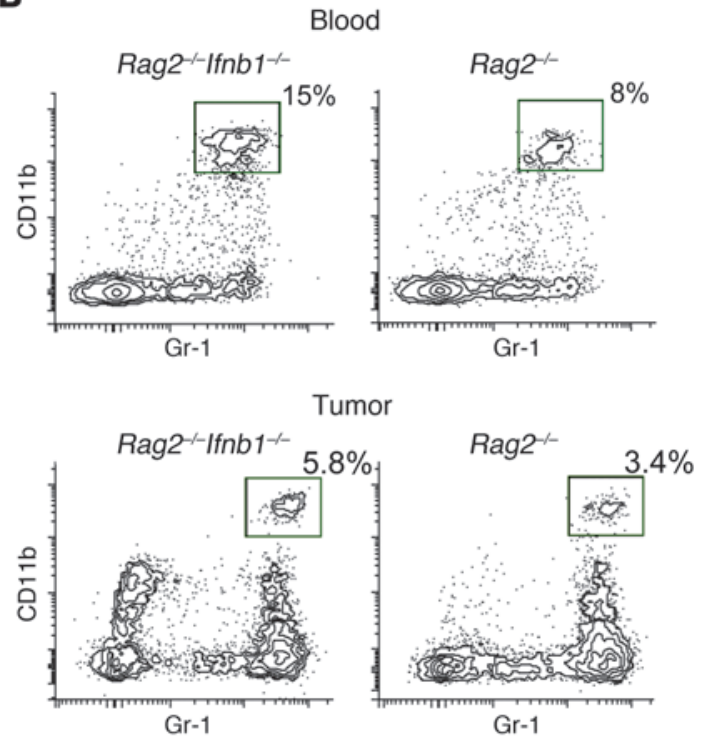

C

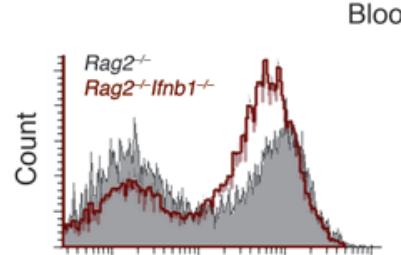

Gr-1
Blood

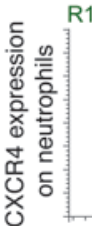

Rag2-

D
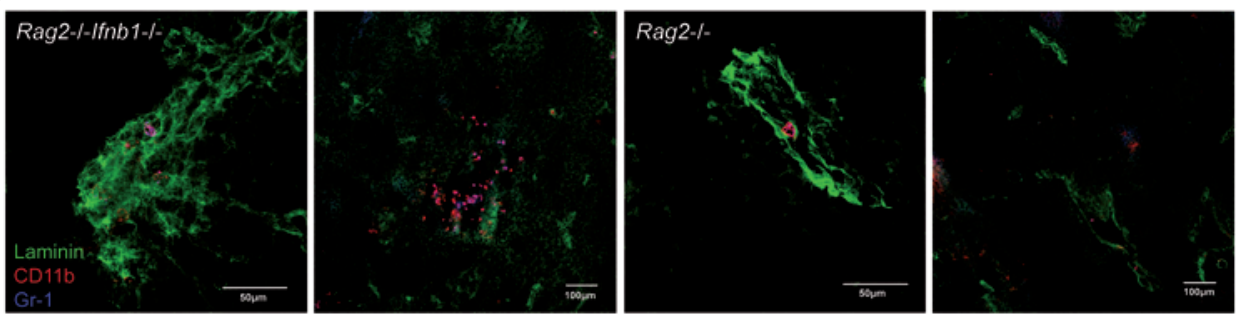

E
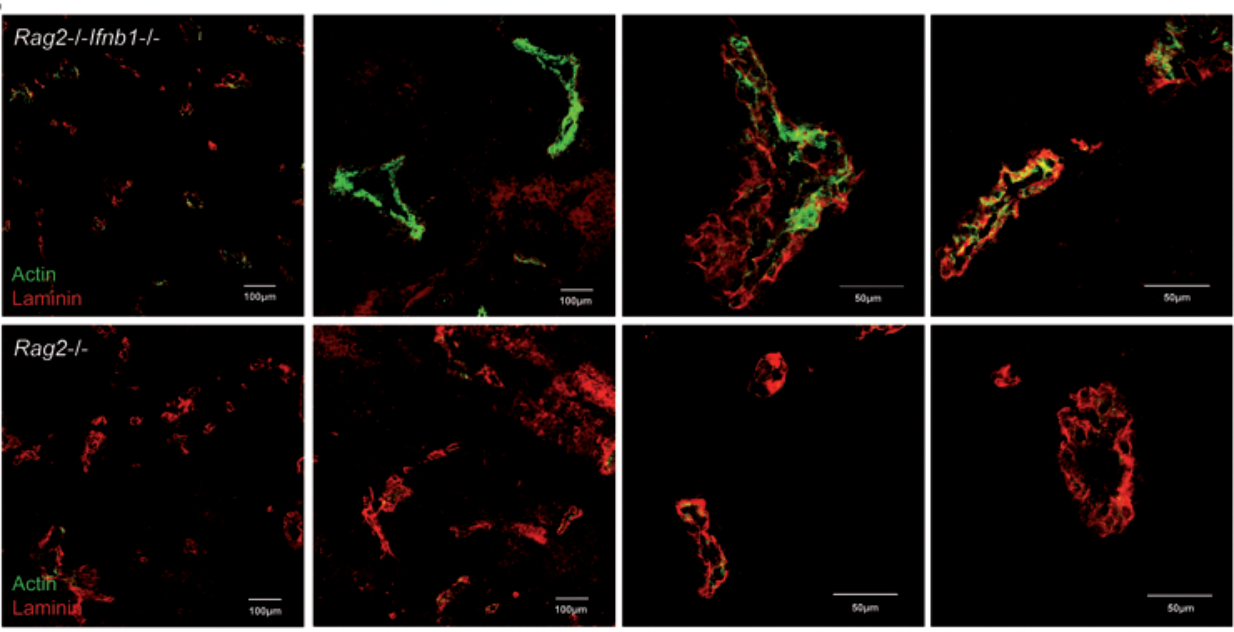

\section{Figure 6}

Restriction of tumor growth does not depend on $T$ and $B$ lymphocytes. (A) Tumor growth in Rag2-/mice is reduced compared with that

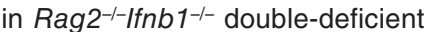
mice. Tumor growth was monitored 14 days, and after this time, tumors removed. (B and $\mathbf{C}$ ) Percentage and number of CD11 $\mathrm{b}^{+}$ $\mathrm{Gr} 1^{+}$neutrophils in blood and infiltrating tumors are higher in Rag2 ${ }^{-/-}$ Ifn $b^{-/-}$mice. CXCR4 expression on blood neutrophils ( $R 1$ gate) is significantly increased in Rag2-/Ifnb $1^{-/-}$mice, which has an impact on their migration into tumor. (D) Immunohistochemistry of tumors

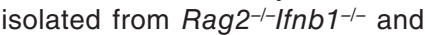
Rag2 $^{-/}$mice shows higher number of infiltrating neutrophils in Rag2 ${ }^{-/-}$ Ifn $b^{-/}$mice compared with Rag2 ${ }^{-/}$ animals. (E) Immunohistochemistry of tumors isolated from Rag2-/Ifnb1/-- and $\mathrm{Rag}^{-/-}$mice shows advanced angiogenic processes in Rag2--Ifnb1-/- mice compared with Rag2 $^{-1-}$ animals. All experiments were carried out as above and done 3 times with at least 5 animals per group. Data represent mean \pm SEM ${ }^{*} P \leq 0.01$ 


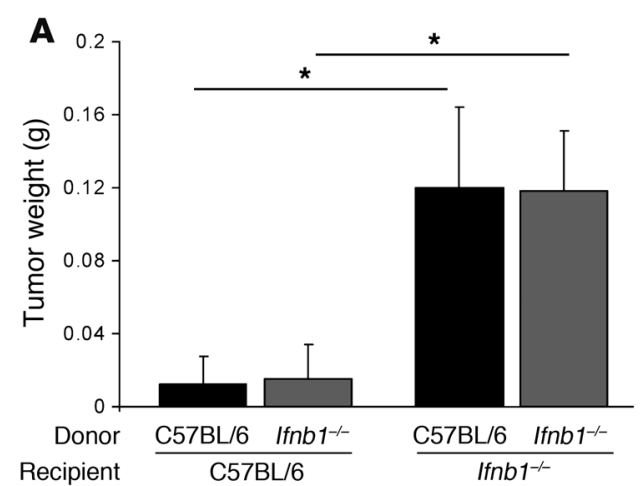

B
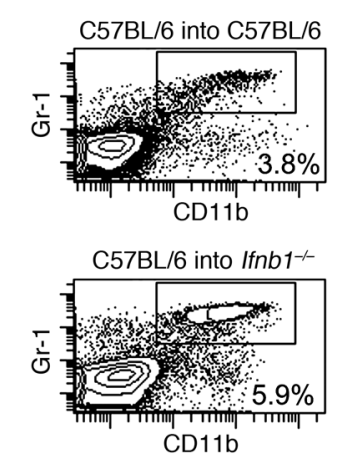

C
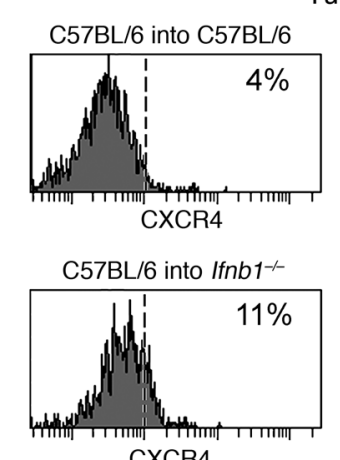

CXCR4
Blood
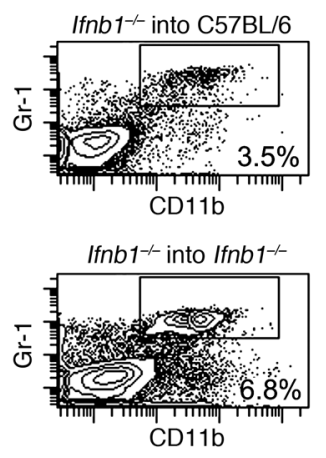

Tumor
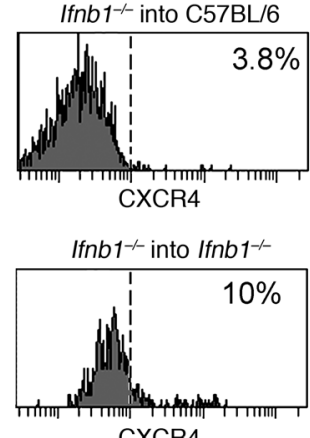

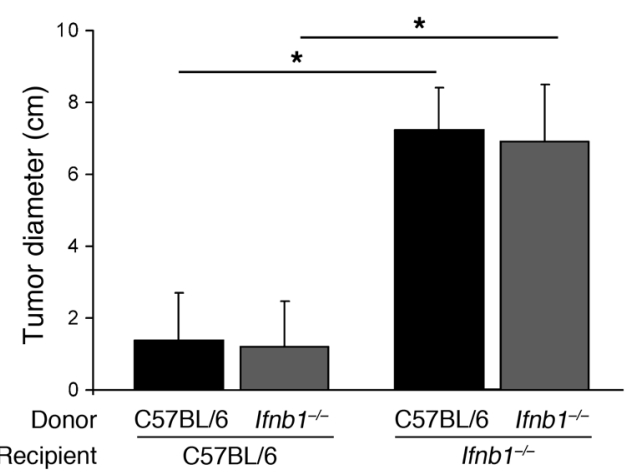

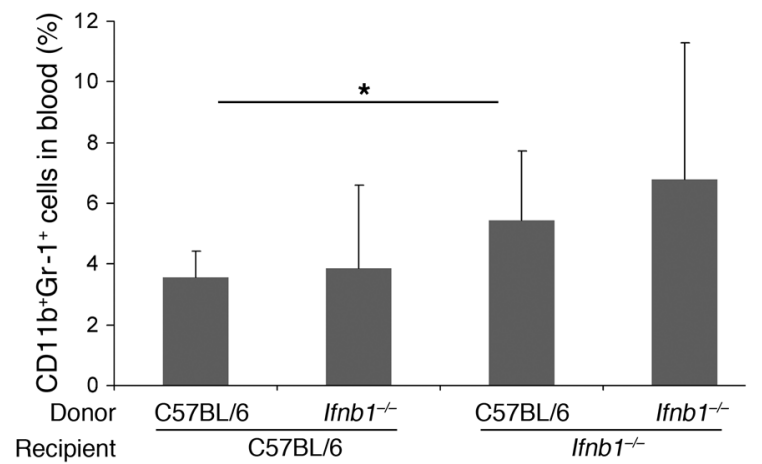

Figure 7

IFN- $\beta$-producing cells are of radio-resistant, nonhematopoietic origin. Recipient Ifnb $1^{-/-}$and C57BL/6 mice were lethally irradiated and reconstituted with bone marrow from donor Ifnb1/-- or C57BL/6 mice. After 6 weeks, chimeras were injected s.c. with B16F10 melanoma cells. Tumors were removed at day 14 and analyzed. (A) Weight and diameter of isolated tumors. (B) Percentage of CD11b+Gr1+ neutrophils in blood. (C) Percentage of $\mathrm{CXCR} 4^{+}$cells in tumor-infiltrating $\mathrm{CD} 11 \mathrm{~b}+\mathrm{Gr} 1^{+}$myeloid cell population. Experiments were done twice with at least 5 animals per group. Data represent mean \pm SEM. ${ }^{*} P \leq 0.05$.

to type I IFNs. We therefore conducted depletion of NK cells in

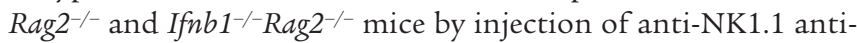
bodies and compared growth of B16F10 tumors in such mice with

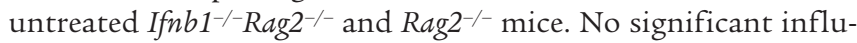
ence of the NK cell depletion on the differential tumor growth could be observed (Supplemental Figure 6).

Endogenous IFN- $\beta$ is produced by radio-resistant, nonbematopoietic cells. The cells responsible for the production of IFN- $\beta$ in steady state are unknown. To obtain a first hint, we wanted to determine whether these cells are of hematopoietic origin. To this end, we lethally irradiated Ifnb $1^{-/-}$and control mice and reconstituted them with bone marrow from either of these mice. Six weeks after transfer, B16F10 melanoma cells were injected s.c., and the tumor size was determined 14 days later. Interestingly, tumors grew stronger in Ifn $b^{-/}$recipient mice, independent of the source of bone marrow (Figure 7A). Tumors in normal recipient mice were not only smaller (Figure 7A) but also exhibited reduced vessel formation (data not shown). In addition, higher numbers of $\mathrm{CD} 11 \mathrm{~b}^{+} \mathrm{Gr} 1^{+}$neutrophils could be isolated from blood and tumors of Ifnb $1^{-/-}$recipients (Figure 7B). Similarly, the percentage of CXCR4 ${ }^{+}$neutrophils was higher in tumors isolated from Ifnb1 $1^{-/-}$recipients (Figure $7 \mathrm{C}$ ). Thus, the cells responsible for pro- 


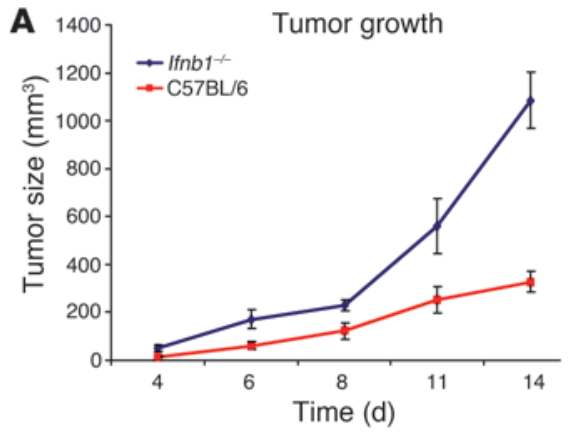

B Blood
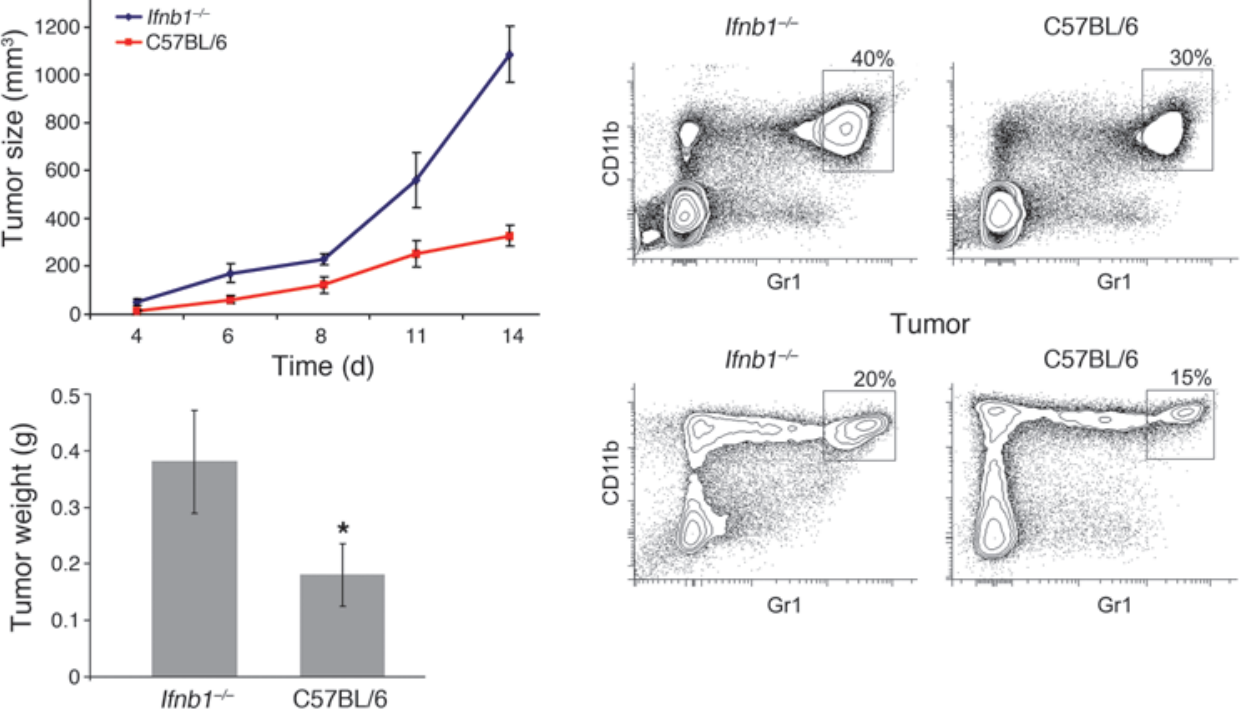

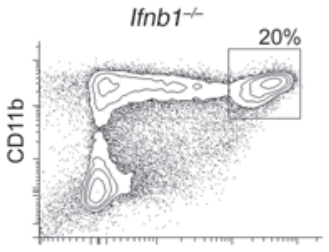

Gr1

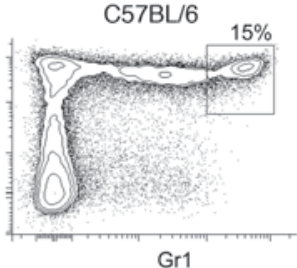

$\mathrm{Gr} 1$
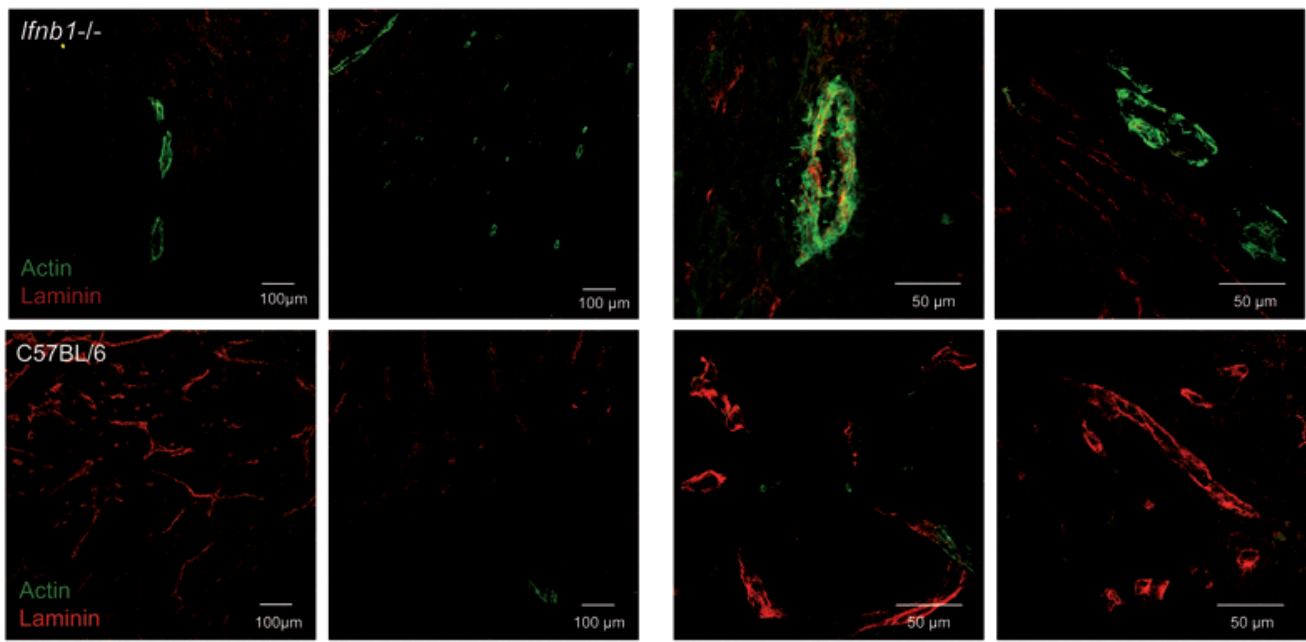

Figure 8

Enhanced MCA205 fibrosarcoma growth and angiogenesis in Ifnb1-/- mice. (A) Growth and size of tumors is significantly higher in Ifnb1 $1^{-/-}$mice. MCA205 fibrosarcoma cells were injected s.c. into the abdomen of C57BL/6 or Ifnb1/- mice, and tumor growth was monitored. At day 14 , mice were sacrificed and tumor weight and diameter were measured. Experiments were done with at least 5 animals per group and repeated at least 3 times with similar results. Data represent mean \pm SEM. ${ }^{*} P \leq 0.01$. (B) Percentage of $C D 11 b+G r 1+$ neutrophils in blood and in Ifnb1-/- tumorbearing mice is higher compared with the control. Tumors were removed at day 14; single-cell solutions prepared, stained, and analyzed using the BD LSR II System. Data were analyzed with FACSDiva software. (C) B16F10 tumors grown in Ifnb1 $1^{-/}$mice exhibit a higher content of fully developed vessels (laminin ${ }^{+} a_{c t i n}+$ ). Histological analysis was done with material collected as described in A, with 10- $\mu \mathrm{m}$ cryosections prepared and stained for laminin (red) and actin (green). Scale bars: $100 \mu \mathrm{m}$ and $50 \mu \mathrm{m}$, respectively. Photographs represent data from at least 3 independent experiments, with at least 3 mice per group.

ducing endogenous IFN- $\beta$ are radio resistant and most likely not of hematopoietic origin.

Inbibition of tumor growth by endogenous IFN- $\beta$ is not specific to B16F10 melanoma. Inhibition of tumor angiogenesis by endogenous IFN- $\beta$ could be peculiar for B16F10. Therefore, we wanted to extend our studies to another tumor model. Cells of the fibrosarcoma MCA205 were injected s.c. into Ifnb1 $1^{-/-}$and WT control mice. Tumor growth was monitored for 14 days (Figure 8A). After 14 days, tumor size and weight were determined. Blood and tumor cells were analyzed by flow cytometry and immunohistology.
Importantly, faster growth of MCA205 tumors was observed in Ifnb1 $1^{-/-}$compared with WT mice, similar to the results obtained for B16F10 melanoma cells. Tumors grew faster and reached significantly larger sizes. Immunohistology showed more advanced tumor angiogenic processes in Ifnb $1^{-/-}$mice compared with the control (Figure 8C), as predicted. Similarly, cytometric analysis of blood and tumor cell samples showed a significantly enhanced neutrophil content in the tumor samples obtained from Ifnb1-/animals (Figure 8B). Thus, retardation of tumor angiogenesis by endogenous IFN- $\beta$ appears to be a general phenomenon. 


\section{Discussion}

Type I IFN system influences carcinogenesis and tumor growth $(59,60)$ and is used as a therapeutic agent for several tumors $(11$, $61)$. One of the reasons for this phenomenon could be that endogenous IFN- $\beta$ affects tumor angiogenesis. The role of IFN- $\beta$ in this event became obvious when B16F10 tumors were transplanted s.c. into mice that were deficient for this cytokine. Tumors grew faster and reached significantly larger size in Ifn $b 1^{-/-}$mice compared with controls, which correlated with enhanced angiogenic processes in the tumor, resulting in more and better-developed blood vessels.

Enhanced tumor growth and angiogenesis in Ifnb1 $1^{-/}$mice appeared to be caused by $\mathrm{CD} 11 \mathrm{~b}^{+} \mathrm{Gr} 1^{+}$neutrophils. Notably, more of these cells could be found in blood, in tumors, and to a small extent already in the bone marrow of such mice. Depletion of neutrophils significantly reduced tumor growth to equal levels in both types of mice, thus abolishing the differential effect of IFN- $\beta$ deficiency. These findings confirm the involvement of $\mathrm{CD} 11 \mathrm{~b}^{+} \mathrm{Gr} 1^{+}$neutrophils in the induction and maintenance of tumor angiogenesis.

Further corroboration of the essential role of neutrophils in tumor angiogenesis was obtained by coinjecting neutrophils and tumor cells. Tumors coinjected with WT neutrophils grew faster than tumor cells alone. However, a dramatic increase in growth rate was observed when the neutrophils could not respond to endogenous type I IFN due to their deficiency in IFNAR. This compellingly shows that neutrophils are the major if not the sole cell population with tumor angiogenic activity, which is strongly controlled by endogenous type I IFN.

The cells that provide endogenous IFN- $\beta$ are radio resistant and therefore most likely of nonhematopoietic origin. Thus, endothelial cells could be one of the sources of IFN- $\beta$. On the other hand, bone marrow stroma cells were also shown to produce type I IFN, hence influencing B cell development (62). These cells could also be responsible for the phenomenon observed here.

In the absence of endogenous IFN- $\beta, \mathrm{CD} 11 \mathrm{~b}^{+} \mathrm{Gr} 1^{+}$neutrophils expressed higher levels of molecules such as CXCR4, VEGF, and MMP9 that are potent factors involved in mobility, tumor homing, and stimulation of angiogenesis (63). In addition, other regulatory molecules that are known to increase tumor homing, such as $c-m y c$ and STAT3 $(44,45,64)$, were also found at significantly higher expression levels in $\mathrm{CD} 11 \mathrm{~b}^{+} \mathrm{Gr} 1^{+}$neutrophils from tumors of mice that lack IFN.

A direct effect of IFN- $\beta$ on the regulation of tumor homing and angiogenesis molecules could indeed be observed in vitro. When $\mathrm{CD} 11 \mathrm{~b}^{+} \mathrm{Gr} 1^{+}$neutrophils from tumors of Ifn $b 1^{-/-}$mice were exposed to low levels of recombinant IFN- $\beta$, genes such as Vegf, Mmp9, and Cxcr4 and their molecular regulators were downregulated to levels found in controls. This is consistent with the hypothesis that low levels of IFN- $\beta$ are constitutively produced by cells like stroma cells (65). Such cells may imprint neutrophils to inhibit tumor angiogenesis. Although only small differences in CXCR 4 expression on $\mathrm{CD} 11 \mathrm{~b}^{+} \mathrm{Gr} 1^{+}$neutrophils could be observed in bone marrow of If $\mathrm{bl}^{-/-}$compared with normal mice, it is possible that such cells immediately leave this anatomical niche upon maturation. Hence, differences might not become apparent.

It is generally accepted that angiogenesis is augmented by hypoxia. Hypoxic regions can be found in tumors of a certain size. Therefore, one could argue that the lack of IFN- $\beta$ leads to a faster initial growth of tumors. This would result in larger hypoxic regions and in turn would induce enhanced angiogenesis. Enhancement of angiogenesis in tumors of mice that lack IFN- $\beta$ would thus result from the initial increased tumor growth and development of large hypoxic areas and would therefore not be due to inhibition of angiogenesis by endogenous IFN- $\beta$. We think we can exclude this argument: (a) tumors of comparable size from both types of mice with presumably similar hypoxic regions were compared. Nevertheless differential angiogenesis was observed. (b) A direct effect of IFN- $\beta$ on molecules involved in angiogenesis induction by $\mathrm{CD} 11 \mathrm{~b}^{+} \mathrm{Gr} 1^{+}$neutrophils could be demonstrated. (c) Coinjection of neutrophils resulted in a dramatic increase in tumor growth rate. At the time of tumor injection, necrosis and hypoxia could not have been different for both groups of tumors. (d) Augmented metastasis formation in mice lacking endogenous IFN- $\beta$ could also be shown, as well as increased angiogenesis in the Matrigel model. These conditions should be identical in both mice, deficient or sufficient for IFN- $\beta$. No difference in hypoxia should exist either in the lungs or in Matrigel pads. Nevertheless, increased metastasis growth and angiogenesis is observed in Ifnb1 $1^{-/-}$mice. Thus, we believe that enhanced growth of tumors in Ifnb1 $1^{-/-}$mice is indeed due to a direct effect of IFN- $\beta$ produced by radio-resistant cells, such as bone marrow stroma cells, on migratory and maturation capacities of $\mathrm{CD} 11 \mathrm{~b}^{+} \mathrm{Gr} 1^{+}$neutrophils that are involved in tumor angiogenesis.

This interpretation is complementary to recent findings on tumor editing by type I IFNs (22). Tumor editing by type I IFNs was shown to act on hematopoietic cells. It was believed that this is due to direct effects on T cells. On the other hand, only part of the tumors induced in mice that lacked a functional type I IFN system were highly immunogenic, i.e., had escaped immune editing by IFN. Here, we describe the complementing explanation. We interpret these findings now to mean that some of the tumors could establish themselves due to the uncontrolled angiogenesis in the absence of an endogenous type I IFN system. The differential growth rates of B16F10 in RAG2-deficient and RAG2 IFN- $\beta$ double-deficient mice are in line with our interpretation. Thus, we conclude that type I IFN could either support an adaptive T cell-dependent tumor immune defense or block tumor angiogenesis via an innate mechanism.

Inhibition of angiogenesis by type I IFNs has been suggested before (66). Many patients suffering from SLE also exhibit vascular diseases such as premature atherosclerosis $(67,68)$. This has been attributed to high amounts of type I IFNs in the serum of such patients. In vitro experiments confirmed the antiangiogenesis potential of type I IFNs. However, this phenotype is observed under inflammatory conditions, with high amounts of IFNs present. In contrast, in a tumor situation, inflammation should be significantly lower than in SLE. Consistently, downregulation of proangiogenic molecules required low to very low amounts of IFN- $\beta$ (data not shown).

Certainly, one could argue that the transplantation of the tumor also initiates an inflammatory reaction. To avoid this problem, we carried out the analysis of tumor-infiltrating $\mathrm{CD} 11 \mathrm{~b}^{+} \mathrm{Gr} 1^{+}$ neutrophils 14 days after tumor implantation. At that time, the inflammatory phase of tumor transplantation should have ceased already. Nevertheless, a clear difference in number and angiogenic properties between neutrophils from control and Ifnb $1^{-/-}$mice could be observed. Therefore, we believe that the low amounts of constitutively produced endogenous IFN- $\beta$ are sufficient to restrict tumor angiogenesis.

Interestingly, no difference was observed in the number of neutrophils in blood of both types of mice not bearing tumors. Possibly the cytokine milieu that is induced in the host organism by the tumor is a prerequisite for the phenotype to become apparent. 
Taken together, our findings demonstrate the importance of the type I IFN system in biological processes apart from its essential role in host defense. Similar to other processes of host response, in which the type I IFN system is involved, the activity of IFN- $\beta$ appears to be pleiotropic and highly complex. Simultaneously, our findings underscore the therapeutic potential of this system when applied appropriately.

\section{Methods}

Tumor cell lines and animals. Melanoma B16F10 tumor cells were used in all experiments. Tumor cell line was purchased from ATCC. As complementary MCA 205 fibrosarcoma cell line was used, kindly provided by T. Blankenstein (Max Delbrück Center for Molecular Medicine, Berlin, Germany). Cells were maintained in Iscove's Modified Dulbecco's Medium (IMDM) (Gibco BRL; Invitrogen) supplemented with 10\% (v/v) heat-inactivated FCS (Integro), $250 \mu \mathrm{mol} / 1 \beta$-mercaptoethanol (Serva), and 1\% (v/v) penicillin/ streptomycin (Sigma-Aldrich). Cells were grown in monolayer at $37^{\circ} \mathrm{C}$ in a humidified $\mathrm{CO}_{2}$ incubator. Exponentially growing cells were harvested with $0.05 \%$ trypsin, washed, and suspended in PBS. The number of cells was counted using a Coulter counter (Beckman Coulter).

All experiments were done using 8- to 12 -week-old female mice. Ifnb1-/and Ifnar1 $1^{-/-}$mice were backcrossed onto the C57BL/6 background for more than 15 generations. Mice were kept under specific pathogen-free conditions at the Helmholtz Center for Infection Research animal facility. The studies were reviewed and approved by an ethics committee of the regulatory authorities Landesamt für Verbraucherschutz und Lebensmittelsicherheit of Lower Saxony in Braunschweig.

Antibodies. PE- or APC-conjugated anti-mouse CD11b (eBioscience), PE-Cy7 or Alexa Fluor 647 anti-mouse Ly6G (Gr-1, RB6-8C5 clone; eBioscience), and biotinylated anti-mouse Ly6C (Pharmingen) were used to characterize tumor-infiltrating neutrophils. Since anti-mouse Ly6G mAb (clone RB6-8C5) reacts with mouse Ly-6G and Ly-6C, additional Ly-6C staining was performed to differentiate between single- and doublepositive cells and to distinguish tumor-infiltrating neutrophils from monocytes (Supplemental Figure 1).

The following antibodies were used: Alexa Fluor 488 anti-mouse CD68 (Serotec), FITC anti-mouse F4/80 (eBioscience), FITC-conjugated antimouse CD14 (eBioscience), PE-conjugated anti-mouse CXCR4 (eBioscience), FITC anti-mouse $\alpha$ SMA (Sigma-Aldrich), anti-mouse laminin $\gamma 1$ chain (Immundiagnostik), Cy3 or Alexa Fluor 488 anti-rabbit IgG (eBioscience), PE-conjugated anti-mouse NKG2D (eBioscience), and FITC-conjugated anti-mouse NK1.1 (eBioscience). To detect biotin, APC-Cy7-conjugated Streptavidin (Pharmingen) was used. For intracellular staining of phosphorylated STAT3, Pacific Blue anti-mouse STAT3 (pY705) (BD Bioscience) was used.

Primers. RT-PCRs were performed with the following primers: MMP9 (s) 5'-TGTCTGGAGATTCGACTTGAAGTC-3' (as) 5'-TGAGTTCCAGGGCACACCA-3'; CXCR4 (s) 5'-CTGAGAAGCATGACGGACAAGT-3' (as) 5'-TGAGGACACTGCTGTAGAGGTTG-3'; c-myc (s) 5'-GCCCCTAGTGCTGCATGAG-3' (as) 5'-CCACAGACACCACATCAATTTCTT-3' (69); STAT3 (s) 5'-TGCCCATGGCTACCTGTT-3' (as) 5'-GAACCTCCTGGGCTTAGTCC- $3^{\prime}$. To standardize the cDNA, the housekeeping gene Rps 9 was tested with primer pairs: (s) 5'-CTGGACGAGGGCAAGATGAAGC-3'; (as) 5'-TGACGTTGGCGGATGAGCACA-3'.

In vitro rmIFN- $\beta$ treatment. Sorted cells were cultured as monolayers at $2 \times 10^{4}$ cells/well in $200 \mu \mathrm{l}$ IMDM medium supplemented as described above. $\mathrm{rmIFN}-\beta$ was added to a concentration of 5 units $/ \mathrm{ml}$. After 4 hours of incubation, cells were harvested and total RNA was extracted using the RNeasy Kit (QIAGEN) according to the manufacturer's protocol. DNA contamination in total RNA was eliminated by incubation with DNaseI
(Amersham Pharmacia Biotech). cDNA was prepared using Superscript II $\mathrm{RNaseH}$ (Invitrogen) according to the manufacturer's instructions.

Murine tumor model. Tumors were initiated by s.c. injection of $10^{5} \mathrm{~B} 16 \mathrm{~F} 10$ melanoma or MCA205 fibrosarcoma cells in $100 \mu \mathrm{l}$ PBS. Tumor sizes were evaluated by caliper every 2 to 3 days. All experiments were done 12 to 14 days after initiation, when maximum tumor diameter was $1.2 \mathrm{~cm}$.

Metastasis formation. Metastases were initiated by i.v. injection of $4 \times 10^{5}$ B16F10 melanoma cells in $100 \mu \mathrm{m}$ PBS. After 14 days, mice were sacrificed; lungs were isolated and analyzed.

In vivo angiogenesis assay. $500 \mu \mathrm{l}$ of $\mathrm{BD}$ Matrigel (BD Bioscience) was injected s.c. into the abdomen of mice. At day 10 , pads were removed and analyzed for hemoglobin content using Drabkin reagent (Sigma-Aldrich).

Neutrophil and NK cell depletion. For depletion of neutrophils, each mouse received i.p. $50 \mu \mathrm{g}$ of monoclonal rat anti-Gr1 antibody (RB6-8C5) in $100 \mu \mathrm{l}$ PBS. Injections were done at day -1 ( 1 day before injection), at day 0 (1 hour before injection), and at days 2, 4, 6, 8, and 10 after tumor cell injection. Depletion was controlled by testing blood samples from treated mice by flow cytometry (Supplemental Figure 1).

NK cells were depleted using rat anti-NK1.1 antibody (RB6-8C5) i.v. $(100 \mu \mathrm{g} /$ mouse). Depletion was conducted twice, at day -2 and day 4 , and controlled as shown in Supplemental Figure 6.

Bone marrow chimeras. Bone marrow from C57BL/6 and Ifnb1 $1^{-/}$mice was isolated (donors) and transferred into both C57BL/ 6 and Ifnb1 $1^{-/-}$lethally irradiated recipients. 6 weeks after transfer, mice were injected s.c. with B16F10 melanoma cells, and tumor growth was measured after 14 days.

Isolation of cells from tumors. Nonnecrotic tumor tissue was cut into 1 to $2 \mathrm{~mm}^{3}$ pieces. The pieces were rinsed twice with PBS and digested using dispase/collagenase A/DNase suspension in IMDM $(0.2 \mathrm{mg} / \mathrm{ml} ; 0.2 \mathrm{mg} / \mathrm{ml}$; $100 \mathrm{mg} / \mathrm{ml}$ ) for 45 minutes in $37^{\circ} \mathrm{C}$. Cell suspension and remaining tissues were then meshed using 50- $\mu \mathrm{m}$ disposable filters (Cell Trics; Partec); subsequently erythrocytes were removed using erythrocyte lysis buffer (ACK buffer). Single-cell suspensions were prepared for FACS staining.

Flow cytometry of the tumor, blood, and bone marrow. Single-cell suspensions from bone marrow were prepared; erythrocytes were removed using erythrocyte lysis buffer (ACK buffer). Single-cell suspensions were prepared for FACS staining.

Erythrocytes of $50 \mu \mathrm{l}$ blood were lysed in $1.5 \mathrm{ml}$ ACK buffer, vortexed, incubated for 5 minutes at room temperature, and centrifuged for 5 minutes. This procedure was repeated once. Then cells were washed once with PBS and prepared for staining.

Single-cell suspensions were stained in FACS buffer with conjugated antibodies listed above, and flow cytometry was performed using a BD LSR II (BD Bioscience). Data were analyzed with BD FACSDiva software (BD Bioscience). Intracellular staining of STAT3 was done using BD Cytofix/ Cytoperm Kit (BD Bioscience) following the manufacturer's manual.

Sorting of the tumor neutrophils. Single-cell suspensions were stained as described for flow cytometry; $\mathrm{CD} 11 \mathrm{~b}^{+} \mathrm{Gr}-1^{+}$neutrophils were sorted using a FACSAria cell sorter (BD Bioscience), and the purity of obtained cells was proved (Supplemental Figure 7).

Coinjection of tumor cells with sorted neutrophils. CD $11 \mathrm{~b}^{+} \mathrm{Gr}-1^{+}$neutrophils from blood and tumors of tumor-bearing Ifnar $1^{--}$and WT mice were sorted using a FACSAria cell sorter $\left(1 \times 10^{5} /\right.$ mouse $)$. Cells were mixed with B16F10 cells $\left(1 \times 10^{5} /\right.$ mouse $)$ and injected s.c. into C57BL $/ 6$ mice. At day 2 and 4 , additional neutrophils were sorted and injected i.v. Tumor growth was monitored. At 14 days mice, were sacrificed and tumor weight was determined. Tumors were snap-frozen and used for histological analysis.

Immunohistochemistry. For immunohistochemical diagnosis and assessment of tumor tissue and vascularization, tumors were dissected, embedded in Tissue-Tek (Sacura), and snap frozen in liquid nitrogen. $7-\mu \mathrm{m}$ cryosections were fixed in acetone at $-20^{\circ} \mathrm{C}$ and air-dried for 
30 minutes. Slides were blocked with $0.05 \%$ BSA in PBS and stained with appropriate antibodies. After staining and washing, slides were dried, mounted with Neo-Mount (Merck), and analyzed using a laser scanning confocal microscope.

Confocal microscopy. Three-color confocal microscopy (FITC/Alexa Fluor 488, PE/Alexa Fluor 568, APC/Cy5/Alexa Fluor 647) of cryosections was performed using LSM 510 META (Zeiss). To avoid overlapping emissions, fluorescent dyes were selectively excited in 2 series and fluorescence of single channels was measured by photon counting. Images were processed with LSM 5 (Zeiss) and Adobe Photoshop 7.

Quantitative analyses of vessels and cells were done using ImageJ Software (http://rsbweb.nih.gov/ij/) and LSM 5 Image Browser. In this context, at least 9 randomly selected vision fields of each tumor were analyzed.

Cytokine and chemokine estimation in tumors. Tumor pieces isolated from $\mathrm{C} 57 \mathrm{BL} / 6$ and $\mathrm{Ifnb}^{-/-}$mice were weighted, homogenized in PBS, and centrifuged, and supernatants were frozen for cytokine and chemokine measurements. For CXCL12 assay, content in tumors was measured using Quantikine (R\&D Systems) following the manufacturer's manual.

For IL-6 and CCL2 assays, content in tumors was measured using BD CBA Mouse Inflammation Kit, following the manufacturer's manual.
Statistics. Values are expressed as mean \pm SEM. Statistical comparisons were made using a 1-tailed Student's $t$ test for single-value comparisons, 1-way ANOVA followed by Dunnett's or Bonferroni's Multiple Comparison Test post analysis, or 2-way ANOVA for comparisons of 3 or more groups (i.e., neutrophil depletion analysis). $P<0.05$ was considered significant.

\section{Acknowledgments}

We wish to thank Regina Lesch and Susanne zur Lage for expert technical assistance. We also thank Nuno Viegas for providing us with statistical analysis. This work was partially supported by grants of the Deutsche Krebshilfe, the Bundesministerium für Bildung und Forschung, and the Deutsche Forschungsgemeinschaft.

Received for publication June 16, 2009, and accepted in revised form January 13, 2010.

Address correspondence to: Jadwiga Jablonska, Helmholtz Centre for Infection Research, HZI, Inhoffenstr. 7, D-38124 Braunschweig, Germany. Phone: 4953161815110; Fax: 4953161815002 ; E-mail: jja@gbf.de.
1. Folkman J. What is the evidence that tumors are angiogenesis dependent? J Natl Cancer Inst. 1990; 82(1):4-6.

2. Plate KH, Breier G, Risau W. Molecular mechanisms of developmental and tumor angiogenesis. Brain Pathol. 1994;4(3):207-218.

3. Auguste P, Lemiere S, Larrieu-Lahargue F, Bikfalvi A. Molecular mechanisms of tumor vascularization. Crit Rev Oncol Hematol. 2005;54(1):53-61.

4. Folkman J. Angiogenesis in cancer, vascular, rheumatoid and other disease. Nat Med. 1995;1(1):27-31.

5. Hanahan D, Weinberg RA. The hallmarks of cancer. Cell. 2000;100(1):57-70.

6. Nyberg P, Xie L, Kalluri R. Endogenous inhibitors of angiogenesis. Cancer Res. 2005;65(10):3967-3979.

7. Phung TL, et al. Pathological angiogenesis is induced by sustained Akt signaling and inhibited by rapamycin. Cancer Cell. 2006;10(2):159-170.

8. Pekarek LA, Starr BA, Toledano AY, Schreiber H. Inhibition of tumor growth by elimination of granulocytes. J Exp Med. 1995;181(1):435-440.

9. Guiducci C, et al. Redirecting in vivo elicited tumor infiltrating macrophages and dendritic cells towards tumor rejection. Cancer Res. 2005; 65(8):3437-3446

10. Nozawa H, Chiu C, Hanahan D. Infiltrating neutrophils mediate the initial angiogenic switch in a mouse model of multistage carcinogenesis. Proc Natl Acad Sci U S A. 2006;103(33):12493-12498.

11. De Palma M, et al. Tumor-targeted interferonalpha delivery by Tie2-expressing monocytes inhibits tumor growth and metastasis. Cancer Cell. 2008;14(4):299-311.

12. Li B, et al. VEGF and PlGF promote adult vasculogenesis by enhancing EPC recruitment and vessel formation at the site of tumor neovascularization. FASEB J. 2006;20(9):1495-1497.

13. Nagase $\mathrm{H}$, et al. Cytokine-mediated regulation of CXCR4 expression in human neutrophils. J Lenkoc Biol. 2002;71(4):711-717.

14. Balkwill $\mathrm{F}$. The significance of cancer cell expression of the chemokine receptor CXCR4. Semin Cancer Biol. 2004;14(3):171-179.

15. Salmaggi A, et al. CXCL12 in malignant glial tumors: a possible role in angiogenesis and crosstalk between endothelial and tumoral cells. J Neurooncol. 2004;67(3):305-317.

16. Zlotnik A. Chemokines and cancer. Int J Cancer. 2006;119(9):2026-2029.

17. Tabatabai G, Frank B, Mohle R, Weller M, Wick W. Irradiation and hypoxia promote homing of hae- matopoietic progenitor cells towards gliomas by TGF-beta-dependent HIF-1alpha-mediated induction of CXCL12. Brain. 2006;129(Pt 9):2426-2435.

18. Samuelsson CV, et al. Transformation of mouse fibroblasts alters the induction pattern of type I IFNs after virus infection. Biochem Biophys Res Commun. 2005;335(2):584-589.

19. Takayanagi $\mathrm{H}$, et al. RANKL maintains bone homeostasis through c-Fos-dependent induction of interferon-beta. Nature. 2002;416(6882):744-749.

20. Koerner I, Kochs G, Kalinke U, Weiss S, Staeheli P. Protective role of beta interferon in host defense against influenza A virus. J Virol. 2007;81(4):2025-2030.

21. Decker T, Muller M, Stockinger S. The yin and yang of type I interferon activity in bacterial infection. Nat Rev Immunol. 2005;5(9):675-687.

22. Dunn GP, Koebel CM, Schreiber RD. Interferons, immunity and cancer immunoediting. Nat Rev Immunol. 2006;6(11):836-848.

23. Deonarain R, et al. Impaired antiviral response and alpha/beta interferon induction in mice lacking beta interferon. J Virol. 2000;74(7):3404-3409.

24. Qin XQ, et al. Interferon-beta gene therapy inhibits tumor formation and causes regression of established tumors in immune-deficient mice. Proc Natl Acad Sci U S A. 1998;95(24):14411-14416.

25. Brassard DL, Grace MJ, Bordens RW. Interferonalpha as an immunotherapeutic protein. J Lenkoc Biol. 2002;71(4):565-581.

26. Ferrantini M, et al. Alpha 1-interferon gene transfer into metastatic Friend leukemia cells abrogated tumorigenicity in immunocompetent mice: antitumor therapy by means of interferon-producing cells. Cancer Res. 1993;53(5):1107-1112.

27. Tuting $T$, et al. Interferon-alpha gene therapy for cancer: retroviral transduction of fibroblasts and particle-mediated transfection of tumor cells are both effective strategies for gene delivery in murine tumor models. Gene Ther. 1997;4(10):1053-1060.

28. Tozer GM, Kanthou C, Baguley BC. Disrupting tumour blood vessels. Nat Rev Cancer. 2005; 5(6):423-435.

29. Hanahan D. A flanking attack on cancer. Nat Med. 1998;4(1):13-14

30. Takakura N. Role of hematopoietic lineage cells as accessory components in blood vessel formation. Cancer Sci. 2006;97(7):568-574.

31. Getts DR, et al. Ly6c+ "inflammatory monocytes" are microglial precursors recruited in a pathogenic manner in West Nile virus encephalitis. J Exp Med. 2008;205(10):2319-2337.
32. Toi M, et al. Significance of thymidine phosphorylase as a marker of protumor monocytes in breast cancer. Clin Cancer Res. 1999;5(5):1131-1137.

33. Lin EY, Nguyen AV, Russell RG, Pollard JW. Colony-stimulating factor 1 promotes progression of mammary tumors to malignancy. J Exp Med. 2001;193(6):727-740.

34. Pollard JW. Tumour-educated macrophages promote tumour progression and metastasis. Nat Rev Cancer. 2004;4(1):71-78.

35. Albini A, Tosetti F, Benelli R, Noonan DM. Tumor inflammatory angiogenesis and its chemoprevention. Cancer Res. 2005;65(23):10637-10641.

36. Yang L, et al. Expansion of myeloid immune suppressor $\mathrm{Gr}+\mathrm{CD} 11 \mathrm{~b}+$ cells in tumor-bearing host directly promotes tumor angiogenesis. Cancer Cell. 2004;6(4):409-421.

37. Darash-Yahana $\mathrm{M}$, et al. Role of high expression levels of CXCR4 in tumor growth, vascularization, and metastasis. FASEB J. 2004;18(11):1240-1242.

38. Du R, et al. HIF1alpha induces the recruitment of bone marrow-derived vascular modulatory cells to regulate tumor angiogenesis and invasion. Cancer Cell. 2008;13(3):206-220.

39. Grunewald M, et al. VEGF-induced adult neovascularization: recruitment, retention, and role of accessory cells. Cell. 2006;124(1):175-189.

40. Lyden D, et al. Impaired recruitment of bone-marrow-derived endothelial and hematopoietic precursor cells blocks tumor angiogenesis and growth. Nat Med. 2001;7(11):1194-1201.

41. Jonak GJ, Knight E Jr. Selective reduction of c-myc mRNA in Daudi cells by human beta interferon. Proc Natl Acad Sci U S A. 1984;81(6):1747-1750.

42. Hu X, Bies J, Wolff L. Interferon beta increases c-Myc proteolysis in mouse monocyte/macrophage leukemia cells. Lenk Res. 2005;29(11):1307-1314.

43. Sarkar D, Park ES, Fisher PB. Defining the mechanism by which IFN-beta dowregulates c-myc expression in human melanoma cells: pivotal role for human polynucleotide phosphorylase (hPNPaseold-35). Cell Death Differ. 2006;13(9):1541-1553.

44. Dessauge F, Hilaly S, Baumgartner M, Blumen B, Werling D, Langsley G. c-Myc activation by Theileria parasites promotes survival of infected B-lymphocytes. Oncogene. 2005;24(6):1075-1083.

45. Busillo JM, Benovic JL. Regulation of CXCR4 signaling. Biochim Biophys Acta. 2007;1768(4):952-963.

46. Yuan ZL, Guan YJ, Wang L, Wei W, Kane AB, Chin $Y E$. Central role of the threonine residue within the $\mathrm{p}^{+1}$ loop of receptor tyrosine kinase in STAT3 
constitutive phosphorylation in metastatic cancer cells. Mol Cell Biol. 2004;24(21):9390-9400.

47. Burger M, et al. Small peptide inhibitors of the CXCR4 chemokine receptor (CD184) antagonize the activation, migration, and antiapoptotic responses of CXCL12 in chronic lymphocytic leukemia B cells. Blood. 2005;106(5):1824-1830.

48. Belardelli F, Ferrantini M. Cytokines as a link between innate and adaptive antitumor immunity. Trends Immunol. 2002;23(4):201-208.

49. Karin M, Greten FR. NF-kappaB: linking inflammation and immunity to cancer development and progression. Nat Rev Immunol. 2005;5(10):749-759.

50. Zlotnik A, Yoshie O. Chemokines: a new classification system and their role in immunity. Immunity. 2000;12(2):121-127.

51. Xue ML, et al. A critical role for CCL2 and CCL3 chemokines in the regulation of polymorphonuclear neutrophils recruitment during corneal infection in mice. Immunol Cell Biol. 2007;85(7):525-531.

52. Reichel CA, et al. $\mathrm{Ccl} 2$ and $\mathrm{Ccl} 3$ mediate neutrophil recruitment via induction of protein synthesis and generation of lipid mediators. Arterioscler Thromb Vasc Biol. 2009;29(11):1787-1793.

53. Reichel CA, et al. Chemokine receptors Ccr1, Ccr2, and $\mathrm{Ccr} 5$ mediate neutrophil migration to postischemic tissue. J Leukoc Biol. 2006;79(1):114-122.

54. Maus $U$, et al. The role of CC chemokine receptor 2 in alveolar monocyte and neutrophil immigra- tion in intact mice. Am J Respir Crit Care Med. 2002; 166(3):268-273.

55. De Keulenaer GW, et al. Convergence of redox-sensitive and mitogen-activated protein kinase signaling pathways in tumor necrosis factor-alpha-mediated monocyte chemoattractant protein-1 induction in vascular smooth muscle cells. Arterioscler Thromb Vasc Biol. 2000;20(2):385-391.

56. Iseki A, et al. Pyrrolidine dithiocarbamate inhibits TNF-alpha-dependent activation of NF-kappaB by increasing intracellular copper level in human aortic smooth muscle cells. Biochem Biophys Res Commun. 2000;276(1):88-92.

57 . Asensi $\mathrm{V}$, et al. In vivo interleukin- 6 protects neutrophils from apoptosis in osteomyelitis. Infect Immun. 2004;72(7):3823-3828.

58. Walker F, et al. IL6/sIL6R complex contributes to emergency granulopoietic responses in G-CSF- and GM-CSF-deficient mice. Blood. 2008; 111(8):3978-3985.

59. Deonarain R, et al. Critical roles for IFN-beta in lymphoid development, myelopoiesis, and tumor development: links to tumor necrosis factor alpha. Proc Natl Acad Sci U S A. 2003;100(23):13453-13458.

60. Gresser I, Belardelli F. Endogenous type I interferons as a defense against tumors. Cytokine Growth Factor Rev. 2002;13(2):111-118.

61. Jonasch E, Haluska FG. Interferon in oncological practice: review of interferon biology, clinical appli- cations, and toxicities. Oncologist. 2001;6(1):34-55.

62. Wang J, Lin Q, Langston H, Cooper MD. Resident bone marrow macrophages produce type 1 interferons that can selectively inhibit interleukin-7driven growth of B lineage cells. Immunity. 1995; 3(4):475-484.

63. Heissig B, et al. Recruitment of stem and progenitor cells from the bone marrow niche requires MMP-9 mediated release of kit-ligand. Cell. 2002;109(5):625-637.

64. Jung JE, et al. STAT3 is a potential modulator of HIF-1-mediated VEGF expression in human renal carcinoma cells. FASEB J. 2005;19(10):1296-1298.

65. Taniguchi T, Takaoka A. A weak signal for strong responses: interferon-alpha/beta revisited. Nat Rev Mol Cell Biol. 2001;2(5):378-386.

66. Denny MF, et al. Interferon-\{alpha\} promotes abnormal vasculogenesis in lupus: a potential pathway for premature atherosclerosis. Blood. 2007; 110(8):2907-2915.

67. Asanuma Y, et al. Premature coronary-artery atherosclerosis in systemic lupus erythematosus. N Engl J Med. 2003;349(25):2407-2415.

68. Roman MJ, et al. Prevalence and correlates of accelerated atherosclerosis in systemic lupus erythematosus. N Engl J Med. 2003;349(25):2399-2406.

69. Chanprasert S, et al. Thrombopoietin (TPO) induces c-myc expression through a. Cell Signal. 2006; 18(8):1212-1218 